\title{
Asymptotic Justification of the \\ Conserved Phase-Field Model with Memory
}

\author{
V. Felli
}

\begin{abstract}
We consider a conserved phase-field model with memory in which the Fourier heat conduction law is replaced by a constitutive assumption of Gurtin-Pipkin type; the system is conserved in the sense that the initial mass of the order parameter is preserved during the evolution. We investigate a Cauchy-Neumann problem for this model which couples an integro-differential equation with a nonlinear fourth-order equation for the phase field. Here we assume that the heat flux memory kernel has a decreasing exponential as principal part and we study the behaviour of solutions when this kernel converges to a Dirac mass. We show that the solution to the conserved phase-field model with memory converges to a solution to the phase-field problem without memory under suitable assumptions on the data.
\end{abstract}

Keywords: Phase-field models, phase transitions, heat conduction with memory, asymptotic analysis, error estimates

AMS subject classification: $35 \mathrm{R} 99,45 \mathrm{~K} 05,80 \mathrm{~A} 22$

\section{Introduction}

A material subject to variation of temperature and phase-transitions occupies an open bounded connected domain $\Omega \subset \mathbb{R}^{N} .(N=1,2,3)$ with smooth boundary $\partial \Omega$, in a given time interval $[0, T] \quad(T>0)$. We assume that only two phases are observed. The dynamic of the system can be characterized by two state variables, namely the relative temperature $\vartheta$ (fixed in order that $\vartheta=0$ is the equilibrium temperature between the two phases) and the phase field $\chi$ (which may stand for the local proportion of one of the two phases).

The evolution of $\vartheta$ and $\chi$ is governed by the differential model

$$
\begin{aligned}
\partial_{t}(\vartheta+\ell \chi)-k_{0} \Delta \vartheta & =g_{0} \\
\partial_{\imath \chi}-\Delta\left(-\Delta \chi+\chi^{3}-\chi-\ell \vartheta\right) & =0
\end{aligned} \quad \text { in } \Omega \times(0, T)
$$

where $g_{0}$ stands for the heat source, $k_{0}$ is a positive constant and $\ell \in \mathbb{R}^{+}$represents the latent heat. We associate with (1.1) the Neumann boundary conditions

$$
k_{0} \partial_{\nu} \vartheta=\partial_{\nu} \chi=\partial_{\nu}\left(-\Delta \chi+\chi^{3}-\chi-\ell \vartheta\right)=0 \quad \text { on } \partial \Omega \times(0, T)
$$

V. Felli: S.I.S.S.A./I.S.A.S., Via Beirut 2-4, 34014 Trieste, Italy; felli@sissa.it 
where $\partial_{\nu}$ denotes the outward normal partial derivative on $\partial \Omega$, and the initial conditions

$$
\left.\begin{array}{l}
\vartheta(0)=\vartheta_{0} \\
\chi(0)=\chi_{0}
\end{array}\right\} \quad \text { in } \Omega
$$

where $\vartheta_{0}$ and $\chi_{0}$ are the initial data for the temperature and phase field, respectively.

In this paper we study some connections between problem (1.1)-(1.3) and the phasefield model analyzed in [9], by developing techniques which are similar to those employed in [7] for the non-conserved phase-field models introduced and analyzed in $[5,6]$. For some of our results one can also see [10].

We notice that $(1.1)_{1}$ is a balance of energy where the diffusion term $k_{0} \Delta \vartheta$ comes out from the Fourier law.

Let us assume the Gurtin-Pipkin law (see [11]) according to which the heat flux $\mathbf{q}$ is given by

$$
\mathbf{q}(x, t)=-\int_{-\infty}^{t} k(t-s) \nabla \vartheta(x, s) d s
$$

so that $\mathbf{q}$ depends only on the temporal history of the temperature gradient $\nabla \vartheta$. In (1.4) $k:(0, T) \rightarrow \mathbb{R}$ represents a heat relaxation kernel. Consequently, under this assumption, the balance of energy is given by

$$
\partial_{\ell}(\vartheta+\ell \chi)-k * \Delta \vartheta=g
$$

where * denotes the time convolution product over $(0, T)$, i.e. for $a$ and $b$ summable in $(0, T)$

$$
(a * b)(t)=\int_{0}^{t} a(s) b(t-s) d s \quad(t \in[0, T]) .
$$

The right-hand side $g$ of equation (1.5) takes not only $g_{0}$ but also the assumption that the past history of the system is known up to $t=0$ into account. Letting $\delta$ stand for the Dirac mass located at $t=0$, we remark that $k_{0} \Delta \vartheta$ may be equivalently set as $k_{0} \delta * \Delta \vartheta$. Arguing as in [7], we take $k=k_{\varepsilon}$ for a suitable sequence $\left\{k_{\varepsilon}\right\}_{\varepsilon>0}$ approximating $k_{0} \delta$ and study the problem with memory corresponding to the kernels $k_{\varepsilon}$, in order to discuss the convergence of the solution to the $\varepsilon$-problem (with memory) to the solution to the limit problem (1.1) - (1.3) (without memory) as $\varepsilon \downarrow 0$.

As far as the memory kernels are concerned, we assume them to be given by the sum of a decreasing exponential

$$
\frac{k_{0}}{\varepsilon} e^{-\frac{t}{\varepsilon}} \quad(t>0)
$$

the trivial extension of which converges to $k_{0} \delta$ in the sense of distributions, and a perturbation converging to 0 in a suitable topology.

Referring to [7], we remark that this choice is motivated by the fact that, if $k_{\varepsilon}$ is given by (1.6), then the heat flux constitutive assumption

$$
\mathbf{q}(x, t)=-\frac{k_{0}}{\varepsilon} \int_{-\infty}^{t} e^{-\frac{t-\vartheta}{\varphi}} \nabla \vartheta(x, s) d s=\mathbf{q}(x, 0)-\left(k_{\varepsilon} * \nabla \vartheta\right)(t)
$$


comes from the well-known Maxwell-Cattaneo law (cf. [4])

$$
\varepsilon \partial_{t} \mathbf{q}+\mathbf{q}=-k_{0} \nabla \vartheta
$$

where $\varepsilon>0$ is small (for $\varepsilon=0$ we have the Fourier law). Setting $w=1 *(\vartheta+\ell \chi)$ and rewriting (1.5) in the form

$$
\partial_{t}^{2} w-k * \Delta\left(\partial_{t} w-\ell \chi\right)=g
$$

i.e.

$$
\partial_{t}^{2} w-k(0) \Delta w=g+k^{\prime} * \Delta w-\ell k * \Delta \chi,
$$

we underline the hyperbolic character of (1.5), since the left-hand side of the previous equation is the wave operator if $k(0)>0$ and $k^{\prime} * \Delta w$ can be considered as a lower order term.

Now we are going to develop three sections. In Section 2, we give an explanation of our notation, the rigorous formulation of the problem, and the statement of our main results. In Section 3; we prove the convergence of the solution to the problern with memory to a solution to the limit problem. In the last, Section 4 , we prove error estimates with respect to the parameter $\varepsilon$.

\section{Main results}

For the sake of convenience, we recall the same notation adopted in [9]. We set

$$
\begin{aligned}
H & =L^{2}(\Omega) \\
V & =H^{1}(\Omega) \\
W & =\left\{v \in H^{2}(\Omega): \partial_{\nu} v=0 \text { on } \partial \Omega\right\}
\end{aligned}
$$

and introduce the operator $A \in \mathcal{L}\left(V, V^{\prime}\right)$ given by

$$
\langle A u, v\rangle=\int_{\Omega} \nabla u \cdot \nabla v \quad(u, v \in V)
$$

where $\langle\cdot, \cdot\rangle$ stands for the duality pairing between $V^{\prime}$ and $V$. We denote by $(\cdot, \cdot)$ the inner product in $H$ (which is identified with its dual space $H^{\prime}$ ), and by $\langle(\cdot, \cdot\rangle\rangle$ the duality pairing between $W^{\prime}$ and $W$. Let $\mathcal{W}, \mathcal{V}, \mathcal{H}, \mathcal{V}^{\prime}, \mathcal{W}^{\prime}$ be the subspaces of $W, V, H, V^{\prime}, W^{\prime}$, respectively, of null-average elements $v$, that is $\langle\langle v, 1\rangle\rangle=0$.

Now, we define the operator $\mathcal{N}: \mathcal{H} \rightarrow \mathcal{W}$ that maps $v \in \mathcal{H}$ into the unique function $\mathcal{N} v \in \mathcal{W}$ which solves

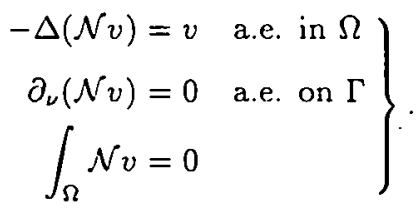


We remark that $\mathcal{N}$ is an isomorphism and can be extended to an isomorphism (still denoted by $\mathcal{N}$ ) from $\mathcal{V}^{\prime}$ to $\mathcal{V}$ given by

$$
\mathcal{N} v \in \mathcal{V}, \quad \int_{\Omega} \nabla(\mathcal{N} v) \cdot \nabla z=\langle v, z\rangle \quad(z \in \mathcal{V})
$$

By transposition, $\mathcal{N}$ is extended to an operator (which is denoted by $\mathcal{N}$ and is an isomorphism again) from $\mathcal{W}^{\prime}$ to $\mathcal{H}$ as

$$
\mathcal{N} v \in \mathcal{H}, \quad-\int_{\Omega}(\mathcal{N} v) \Delta z=\langle\langle v, z\rangle\rangle \quad(z \in \mathcal{W})
$$

Note that the norm

$$
\left(\int_{\Omega}|\nabla(\mathcal{N} v)|^{2}\right)^{\frac{1}{2}}=\langle v, \mathcal{N} v\rangle^{\frac{1}{2}} \quad \text { is equivalent to }\|v\| v^{\prime} \quad\left(v \in \mathcal{V}^{\prime}\right)
$$

and we use this norm instead of $\|v\| v$, whenever it is more convenient. Besides,

$$
\left(\int_{\Omega}|\mathcal{N} v|^{2}\right)^{\frac{1}{2}} \quad \text { is equivalent to }\|v\|_{W^{\prime}} \quad\left(v \in \mathcal{W}^{\prime}\right)
$$

Let us use the same notation $\|\cdot\|_{H}$ both for the norm in $H=L^{2}(\Omega)$ and for that in $H^{N}=L^{2}\left(\Omega, \mathbb{R}^{N}\right)$.

Let us assume

$$
k_{0}, \ell \in(0, \infty)
$$

and let us consider the $\varepsilon$-problem (for all $\varepsilon>0$ )

$$
\left.\begin{array}{rlrl}
\partial_{t}\left(\vartheta_{\varepsilon}+\ell \chi_{\varepsilon}\right)-\Delta\left(k_{\varepsilon} * \vartheta_{\varepsilon}\right) & =g_{\varepsilon} & & \text { in } Q_{T} \\
\partial_{t} \chi_{\varepsilon}-\Delta\left(-\Delta \chi_{\varepsilon}+\chi_{\varepsilon}^{3}-\chi_{\varepsilon}-\ell \vartheta_{\varepsilon}\right) & =0 & & \text { in } Q_{T} \\
\partial_{\nu}\left(k_{\varepsilon} * \vartheta_{\varepsilon}\right)=\partial_{\nu} \chi_{\varepsilon}=\partial_{\nu}\left(-\Delta \chi_{\varepsilon}+\chi_{\varepsilon}^{3}-\chi_{\varepsilon}-\ell \vartheta_{\varepsilon}\right) & =0 & & \text { on } \Sigma_{T} \\
\vartheta_{\varepsilon}(0)=\vartheta_{0, \varepsilon} \text { and } \chi_{\varepsilon}(0) & =\chi_{0, \varepsilon} & & \text { in } \Omega
\end{array}\right\}
$$

where $Q_{T}=\Omega \times(0, T)$ and $\Sigma_{T}=\partial \Omega \times(0, T)$. The kernel $k_{\varepsilon}$ in $(2.5)_{1}$ is the unique solution to the singular perturbation problem

$$
\begin{aligned}
\varepsilon k_{\varepsilon}^{\prime}+k_{\varepsilon} & =\pi_{\varepsilon} \\
k_{\varepsilon}(0) & =\frac{k_{0}}{\varepsilon}
\end{aligned}
$$

that is

$$
k_{\varepsilon}=\frac{k_{0}}{\varepsilon} e^{-\frac{1}{\varepsilon}}+\frac{1}{\varepsilon} e^{-\frac{1}{\epsilon}} * \pi_{\varepsilon}
$$

for a given

$$
\pi_{\varepsilon} \in W^{2,1}(0, T) .
$$


Let $g_{e}$ satisfy the conditions

$$
\begin{aligned}
g_{e} & \in L^{2}(0, T ; H) \cap\left(W^{1,1}(0, T ; H)+W^{2,1}\left(0, T ; V^{\prime}\right)\right) \\
g_{e}(0) & \in H
\end{aligned}
$$

Assume also

$$
\left.\begin{array}{l}
\vartheta_{0, \varepsilon} \in V \\
\chi_{0, c} \in V
\end{array}\right\}
$$

and that the kernels $k_{\varepsilon}$ are of positive type, that is

$$
\int_{0}^{T}\left(v(t),\left(k_{\varepsilon} * v\right)(t)\right) d t \geq 0 \quad\left(v \in L^{2}(0, T ; H), T \in(0,+\infty)\right) .
$$

Note that there is indeed at least one memory kernel given by (2.8) which fulfils (2.13), namely the one obtained for $\pi_{\varepsilon}=0$. Besides, a sufficient condition which guarantees that $k_{\varepsilon}$ is of positive type is that $\pi_{\varepsilon}$ is of positive type, since [13: p. 278/Theorem XX] ensures that the convolution of functions of positive type is of positive type.

A weak formulation of problem (2.5) can be stated as

$$
\left.\begin{array}{rl}
\vartheta_{\varepsilon} & \in W^{1,1}\left(0, T ; W^{\prime}\right) \cap L^{\infty}(0, T ; H) \cap L^{2}(0, T ; V) \\
\chi_{\varepsilon} & \in H^{1}\left(0, T ; V^{\prime}\right) \cap L^{\infty}(0, T ; V) \cap L^{2}(0, T ; W) \\
\ell_{\chi_{\varepsilon}} & \in C^{0}([0, T], V) \cap C^{1}([0, T] ; H) \\
\xi_{\varepsilon} & \in L^{2}(0, T ; V)
\end{array}\right\}
$$

and

$$
\begin{aligned}
& \left\langle\left\langle\partial_{t}\left(\vartheta_{\varepsilon}+\ell \chi_{\varepsilon}\right)(t), v\right\rangle\right\rangle-\int_{\Omega}\left(k_{\varepsilon} * \vartheta_{\varepsilon}\right)(t) \Delta v=\int_{\Omega} g_{\varepsilon}(t) v \quad(v \in W, \text { a.e. in }(0, T)) \\
& \xi_{\varepsilon}=-\Delta \chi_{\varepsilon}+\chi_{\varepsilon}^{3}-\chi_{\epsilon}-\ell \vartheta_{\varepsilon} \\
& \left\langle\partial_{t} \chi_{\epsilon}(t), v\right\rangle+\int_{\Omega} \nabla \xi_{\varepsilon}(t) \cdot \nabla v=0 \quad(v \in V, \text { a.c. in }(0, T)) \\
& \vartheta_{\varepsilon}(0)=\vartheta_{0, \varepsilon} \text { and } \chi_{\varepsilon}(0)=\chi_{0, \varepsilon} .
\end{aligned}
$$

This has been proved in [12] (existence) and [9] (uniqueness) under weaker assumptions on the data $k_{\varepsilon}, g_{\varepsilon}, \vartheta_{0 \varepsilon}$ and, consequently, with less regularity than that stated above. On the other hand, assuming our hypotheses one can easily obtain regularity (2.14) for the solution to the $\varepsilon$-problem. In particular, to obtain the improved regularity $(2.14)_{3}$ claimed on $\eta_{\varepsilon}$, we set $w_{\varepsilon}=1 * \eta_{\varepsilon}$ and rewrite $(2.15)_{1}$ as

$$
w_{\varepsilon}^{\prime \prime}+k_{\varepsilon}(0) A w_{\varepsilon}=f_{\varepsilon}-A\left(k_{\varepsilon}^{\prime} * w_{\varepsilon}\right)
$$

where $f_{\varepsilon}=g_{\varepsilon}-\ell \Delta\left(k_{\varepsilon} * \chi_{\varepsilon}\right)$, with the initial conditions

$$
\left.\begin{array}{l}
w_{e}(0)=0 \\
w_{\varepsilon}^{\prime}(0)=\eta_{0, \varepsilon}=\vartheta_{0, \varepsilon}+\ell_{\chi_{0, e}}
\end{array}\right\}
$$


Since $f_{\varepsilon} \in L^{1}(0, T ; H)+W^{1,1}\left(0, T ; V^{\prime}\right)$ and $\eta_{0, \varepsilon} \in V$, we can apply [1: p. 62/Theorem 4.4] to get

$$
w_{\varepsilon} \in C^{0}([0, T] ; V) \cap C^{1}([0, T] ; H) \text {. }
$$

Then we set $f_{\varepsilon}^{1}=f_{\varepsilon}-A\left(k_{\varepsilon}^{\prime} * w_{\varepsilon}\right)$, thus we obtain

$$
\left.\begin{array}{rl}
w_{c}^{\prime \prime}+k_{\varepsilon}(0) A w_{\varepsilon} & =f_{\varepsilon}^{1} \\
w_{\varepsilon}(0) & =0 \\
w_{\varepsilon}^{\prime}(0) & =\eta_{0, \varepsilon}
\end{array}\right\} .
$$

Since $f_{\varepsilon}^{1} \in W^{1,1}(0, T ; H)+W^{2,1}\left(0, T ; V^{\prime}\right)$ and $f_{\varepsilon}^{1}(0) \in H$, using [1: p. 74/Theorem 5.1] we have

$$
w_{\varepsilon} \in C^{1}([0, T] ; V) \cap C^{2}([0, T] ; H)
$$

and so $(2.14)_{3}$ is proved.

One can also see $[8]$, where a generalized system is studied and regularity results are presented as well.

Thanks to $(2.14)_{1}, \vartheta_{\epsilon}$ takes values into $V$ so that problem (2.5) can be rewritten by means of the operator $A$ as

$$
\left.\begin{array}{rl}
\partial_{t}\left(\vartheta_{\varepsilon}+\ell \chi_{\varepsilon}\right)+A\left(k_{\varepsilon} * \vartheta_{\varepsilon}\right) & =g_{\varepsilon} \\
\partial_{t} \chi_{\varepsilon}+A \xi_{\varepsilon} & =0 \\
\xi_{\varepsilon} & =A \chi_{\varepsilon}+\chi_{\varepsilon}^{3}-\chi_{\varepsilon}-\ell \vartheta_{\varepsilon} \\
\vartheta_{\varepsilon}(0)=\vartheta_{0, \varepsilon} \quad \text { and } \quad \chi_{\varepsilon}(0) & =\chi_{0, \varepsilon}
\end{array}\right\} \quad\left(\text { in } V^{\prime}, \text { a.e. in }(0, T)\right)
$$

If the unknown function $\vartheta_{\varepsilon}$ is replaced by the integrated enthalpy $w_{\varepsilon}$, namely

$$
w_{\varepsilon}=1 *\left(\vartheta_{\varepsilon}+\ell_{\chi \varepsilon}\right)
$$

problem (2.16) is transformed into

$$
\left.\begin{array}{rl}
\partial_{t}^{2} w_{\varepsilon}+A\left(k_{\varepsilon} * \partial_{t} w_{\varepsilon}\right) & =g_{\varepsilon}+A\left(k_{\varepsilon} * \ell \chi_{\varepsilon}\right) \\
\partial_{t} \chi_{\epsilon}+A \xi_{\varepsilon} & =0 \\
+\left(\ell^{2}-1\right) \chi_{\varepsilon}-\ell \partial_{t} w_{\varepsilon} & =\xi_{\varepsilon} \\
\chi_{\epsilon}(0)=\chi_{0, \varepsilon}, w_{\varepsilon}(0) & =0
\end{array}\right\}
$$

We now consider the limit problem

$$
\begin{aligned}
\partial_{t}(\vartheta+\ell \chi)+k_{0} A \vartheta & =g & & \text { in } W^{\prime} \\
\partial_{t} \chi+A \xi & =0 & & \text { in } V^{\prime} \\
A \chi+\chi^{3}-\chi-\ell \vartheta & =\xi & & \text { in } V^{\prime} \\
\vartheta(0)=\vartheta_{0}, \chi(0) & =\chi_{0} & &
\end{aligned} \quad(\text { for a.e. } t \in(0, T))
$$

Arguing as above, we introduce the integrated enthalpy

$$
w=1 *(\vartheta+\ell \chi)
$$


and write the limit problem in terms of $w$ as

$$
\left.\begin{array}{r}
\partial_{t}^{2} w+k_{0} A\left(\partial_{t} w-\ell_{\chi}\right)=g \\
\partial_{t} \chi+A \xi=0 \\
A \chi+\chi^{3}-\chi-\ell\left(\partial_{t} w-\ell \chi\right)=\xi \\
\partial_{t} w(0)=\vartheta_{0}+\ell \chi_{0}, \chi(0)=\chi_{0}, w(0)=0
\end{array}\right\}
$$

As far as the data are concerned, we assume

$$
\left.\begin{array}{rl}
g & \in L^{2}(0, T ; H) \\
\vartheta_{0} & \in V^{\prime} \\
\chi_{0} & \in H
\end{array}\right\}
$$

and look for a solution $(\vartheta, \chi, \xi)$ to problem (2.19) such that

$$
\left.\begin{array}{l}
\vartheta \in H^{1}\left(0, T ; W^{\prime}\right) \cap L^{\infty}(0, T ; H) \\
\chi \in L^{\infty}(0, T ; V) \cap H^{1}\left(0, T ; V^{\prime}\right) \cap L^{2}(0, T ; W) \\
\xi \in L^{2}(0, T ; V)
\end{array}\right\} .
$$

Now, let us state our results. The first one ensures the convergence of the conserved phase-field model with memory to the conserved phase-field model without memory (classical model). For the uniqueness of the solution to the limit problem we refer to [3: p. 550/Theorem 3.1].

Without any loss of generality, we can assume $\varepsilon<1$ and require boundedness of converging terms to hold for $0<\varepsilon<1$.

Theorem 2.1. For $\varepsilon \in(0,1)$, assume $(2.4),(2.6)-(2.13),(2.22)$ and let $\left(\vartheta_{\varepsilon}, \chi_{\epsilon}, \xi_{\varepsilon}\right)$ be the solution to problem (2.16) satisfying (2.14). Moreover, assume that

$$
\begin{aligned}
\chi_{0, \varepsilon} \rightarrow \chi_{0} \text { in } H, \vartheta_{0, \varepsilon} & \rightarrow \vartheta_{0} \text { in } V^{\prime} \\
g_{\varepsilon} & \rightarrow g \text { in } L^{2}(0, T ; H) \\
\pi_{\varepsilon} & \rightarrow 0 \text { in } W^{1,1}(0, T)
\end{aligned}
$$

as $\varepsilon \downarrow 0$ and that, for any $\varepsilon \in(0,1)$,

$$
\begin{aligned}
& \chi_{\varepsilon} \in C^{0}([0, T] ; V) \\
& \left\|\vartheta_{0, \varepsilon}\right\|_{H}+\left\|\chi_{0, \varepsilon}\right\|_{V}+\left\|g_{\varepsilon}\right\|_{L^{2}(0, T ; H)} \leq c_{0}
\end{aligned}
$$

for some constant $c_{0}>0$. Then, there exists a triplet $(\vartheta, \chi, \xi)$ satisfying $(2.23)$ and such that the strong, weak star and weak convergences

$$
\begin{array}{rlll}
1 * \vartheta_{\varepsilon} \rightarrow 1 * \vartheta & \text { in } & C^{0}([0, T] ; H) \cap H^{1}\left(0, T ; V^{\prime}\right) \\
\chi_{\varepsilon} \rightarrow \chi & \text { in } & C^{0}([0, T] ; H) \cap L^{2}(0, T ; V) \\
\vartheta_{\varepsilon} \doteq \vartheta & \text { in } & L^{\infty}(0, T ; H) \cap H^{1}\left(0, T ; W^{\prime}\right) \\
\chi_{\varepsilon} \doteq \chi & \text { in } & L^{\infty}(0, T ; V) \cap H^{1}\left(0, T ; V^{\prime}\right) \cap L^{2}(0, T ; W) \\
\xi_{\varepsilon}-\xi & \text { in } & L^{2}(0, T ; V) \\
\chi_{e}^{3} * \chi^{3} & \text { in } & L^{\infty}(0, T ; H)
\end{array}
$$


hold. Moreover, the triplet $(\vartheta, \chi, \xi)$ yields the solution to problem (2.19).

Sufficient conditions in order to ensure the validity of (2.27) are given in [8].

We next present an error estimate, for which we ask for additional bounds on the sequences $\left\{g_{\varepsilon}\right\},\left\{\vartheta_{0, \varepsilon}\right\},\left\{\chi_{0, \varepsilon}\right\},\left\{\pi_{\varepsilon}\right\}$, besides (2.28). Let $\left(\vartheta_{\varepsilon}, \chi_{\varepsilon}, \xi_{\varepsilon}\right)$ be the solution to problem (2.16) satisfying $(2.14)$ and $(\vartheta, \chi, \xi)$ the solution to problem (2.19) satisfying (2.23).

Theorem 2.2. In addition to the assumptions of Theorem 2.1, assume

$$
g_{\varepsilon} \in H^{1}(0, T ; H)+W^{2,1}\left(0, T ; V^{\prime}\right)
$$

and

$$
\begin{aligned}
\varepsilon^{\frac{3}{2}}\left\|g_{\varepsilon}(0)\right\|_{H} & +\varepsilon\left\|\vartheta_{0, \varepsilon}+\ell \chi_{0, \varepsilon}\right\| V+\varepsilon\left\|g_{\varepsilon}\right\|_{H^{1}(0, T ; H)+W^{2,1}\left(0, T ; V^{\prime}\right)} \\
+ & \left|\int_{\Omega}\left(\chi_{0, \varepsilon}-\chi_{0}\right)\right|+\left\|\chi_{0, \varepsilon}-\chi_{0}\right\| V^{\prime}+\left\|\vartheta_{0, \varepsilon}-\vartheta_{0}\right\|_{V^{\prime}} \\
& \quad+\left\|\pi_{\varepsilon}\right\|_{W^{1,1}(0, T)}+\left\|g_{\varepsilon}-g\right\|_{L^{2}(0, T ; H)} \leq c_{1} \varepsilon^{\frac{1}{2}}
\end{aligned}
$$

for any $\varepsilon \in(0,1)$ and for some constant $c_{1}>0$. Then the error estimate

$$
\left\|\left(1 * \vartheta_{\varepsilon}\right)-(1 * \vartheta)\right\|_{H^{\prime}(0, T ; H) \cap L^{\infty}(0, T ; V)}+\left\|\chi_{\varepsilon}-\chi\right\|_{L^{\infty}\left(0, T ; V^{\prime}\right) \cap L^{2}(0, T ; V)} \leq C_{1} \varepsilon^{\frac{1}{2}}
$$

holds for any $\varepsilon \in(0,1)$ with $C_{1}>0$ a constant depending only on $\Omega, T, c_{0}, c_{1}, \ell, k_{0}$ and on the upper bounds for the data related to (2.24) - (2.26).

In the following proofs, for the sake of convenience, $c>0$ denotes a constant which may vary from line to line, but it always depends on $\Omega, k_{0}, \ell, T$ and on the data at most. Constants like $B_{1}, B_{2}$ etc. depend only on the data (not on the approximation parameter). We point out that $\sigma>0$ always denotes a parameter which is chosen small enough in each step of the proofs and a symbol like $c_{\sigma}$ is employed to stress the dependance on the parameter $\sigma$.

Moreover, we recall the formulas

$$
\left.\begin{array}{rl}
a * b & =a(0)(1 * b)+a_{t} * 1 * b \\
(a * b)_{t} & =a(0) b+a_{t} * b
\end{array}\right\}
$$

which hold whenever they make sense, and the Young theorem

$$
\|a * b\|_{L^{r}\left(0, T_{;} X\right)} \leq\|a\|_{L^{p}(0, T)}\|b\|_{L^{q}(0, T ; X)}
$$

where $X$ is a real Banach space and $p, q, r \in[1, \infty]$ with $\frac{1}{r}=\frac{1}{p}+\frac{1}{q}-1$. Finally, we use the clementary inequality

$$
2 a b \leq \sigma a^{2}+\frac{1}{\sigma} b^{2} \quad(a, b \geq 0, \sigma>0)
$$

and extended versions of the Gronwall lemma (see [2: pp. 156 - 157]). 


\section{Proof of Theorem 2.1}

The scheme of the proof is the following. First of all we prove some a priori estimates for the solution $\left(\vartheta_{\varepsilon}, \chi_{\epsilon}, \xi_{\varepsilon}\right)$ to problem (2.16). Then we select some converging sequences by weak and weak star compactness. Finally, we show that the limits of these sequences solve problem (2.19).

First set of a priori estimates. The first a priori estimate, which we derive for the reader's convenience, is actually the same inequality deduced in [9: Theorem 2.1]. We sum $(2.16)_{1}$ tested by $\vartheta_{\varepsilon}(t)$ (note that $\vartheta_{\varepsilon}$ takes values into $V$ )

$$
\left\langle\partial_{t} \vartheta_{\varepsilon}(t), \vartheta_{\varepsilon}(t)\right\rangle+\ell\left\langle\partial_{t} \chi_{\varepsilon}(t), \vartheta_{\varepsilon}(t)\right\rangle+\int_{\Omega}\left(k_{\varepsilon} * \nabla \vartheta_{\varepsilon}\right)(t) \cdot \nabla \vartheta_{\varepsilon}(t)=\left\langle g_{\varepsilon}(t), \vartheta_{\varepsilon}(t)\right\rangle
$$

for a.e. $t \in(0, T)$ with $(2.16)_{2}$ tested by $\mathcal{N}\left(\partial_{\imath} \chi_{\varepsilon}\right)(t)$ (note that $\partial_{\imath} \chi_{\varepsilon}(t) \in \mathcal{V}^{\prime}$ by $(2.15)_{3}$ tested by $v=1$ ), i.e.

$$
\left\langle\partial_{t} \chi_{\varepsilon}(t), \mathcal{N}\left(\partial_{t} \chi_{\varepsilon}\right)(t)\right\rangle+\left\langle A \xi_{\varepsilon}(t), \mathcal{N}\left(\partial_{t} \chi_{\varepsilon}(t)\right)\right\rangle=0 \quad \text { a.e. in }(0, T) .
$$

Using (2.2), (2.3) and $(2.16)_{3}$, integrating with respect to time and remarking that

$$
\int_{0}^{t}\left(\int_{\Omega}\left(k_{\varepsilon} * \nabla \vartheta_{\varepsilon}\right)(s) \cdot \nabla \vartheta_{\varepsilon}(s)\right) d s \geq 0 \quad(t>0)
$$

by virtue of (2.13), we infer that

$$
\begin{aligned}
& \frac{1}{2}\left\|\vartheta_{\varepsilon}(t)\right\|_{H}^{2}+\int_{0}^{t}\left\|\partial_{t} \chi_{\varepsilon}(s)\right\|_{V}^{2}, d s+\frac{1}{2}\left\|\nabla \chi_{\varepsilon}(t)\right\|_{H}^{2}+\frac{1}{4} \int_{\Omega}\left(\chi_{\varepsilon}^{2}(t)-1\right)^{2} \\
& \leq \frac{1}{2}\left\|\vartheta_{0, \varepsilon}\right\|_{H}^{2}+\frac{1}{2}\left\|\nabla \chi_{0, \varepsilon}\right\|_{H}^{2}+\frac{1}{4} \int_{\Omega}\left(\chi_{0, \varepsilon}^{2}-1\right)^{2}+\int_{0}^{t}\left(\int_{\Omega} g_{\varepsilon}(s) \vartheta_{\varepsilon}(s)\right) d s .
\end{aligned}
$$

Applying the generalized Gronwall lemma, we obtain

$$
\begin{aligned}
& \left\|\vartheta_{\varepsilon}(t)\right\|_{H}^{2}+\int_{0}^{t}\left\|\partial_{t} \chi_{\varepsilon}(s)\right\|_{V}^{2}, d s+\left\|\nabla \chi_{e}(t)\right\|_{H}^{2}+\int_{\Omega}\left(\chi_{\varepsilon}^{2}(t)-1\right)^{2} \\
& \leq c\left(\left\|\vartheta_{0, \epsilon}\right\|_{H}^{2}+\left\|\nabla \chi_{0, \varepsilon}\right\|_{H}^{2}+\int_{\Omega}\left(\chi_{0, \varepsilon}^{2}-1\right)^{2}+\left(\int_{0}^{t}\left\|g_{\varepsilon}(s)\right\|_{H} d s\right)^{2}\right)
\end{aligned}
$$

with $c>0$ being a constant.

Note that, owing to the continuous embedding $H^{1}(\Omega) \subset L^{4}(\Omega)$,

$$
\int_{\Omega}\left(\chi_{0, \varepsilon}^{2}-1\right)^{2} \leq c\left\|\chi_{0, \varepsilon}\right\|_{V}^{4}+|\Omega|
$$

where $|\cdot|$ is the Lebesgue measure in $\mathbb{R}^{N}$. Hence we find the estimates

$$
\begin{aligned}
& \left\|\vartheta_{\varepsilon}(t)\right\|_{H}^{2}+\int_{0}^{t}\left\|\partial_{t} \chi_{e}(s)\right\|_{V^{\prime}}^{2} d s+\left\|\nabla \chi_{\varepsilon}(t)\right\|_{H}^{2}+\left\|\chi_{e}(t)\right\|_{L^{4}(\Omega)}^{4} \cdot \\
& \quad \leq c\left(\left\|\vartheta_{0, \varepsilon}\right\|_{H}^{2}+\left\|\nabla_{\chi_{0, e}}\right\|_{H}^{2}+\left\|\chi_{0, \varepsilon}\right\|_{V}^{4}+1+\left\|g_{\epsilon}\right\|_{L^{1}(0, T ; H)}^{2}\right) \\
& \quad \leq c\left(\left\|\vartheta_{0, \varepsilon}\right\|_{H}^{2}+1+\left\|\chi_{0, \varepsilon}\right\|_{V}^{4}+\left\|g_{e}\right\|_{L^{1}(0, T ; H)}^{2}\right) \\
& \quad=c B(\varepsilon)
\end{aligned}
$$


where

$$
B(\varepsilon)=\left\|\vartheta_{0, \varepsilon}\right\|_{H}^{2}+1+\left\|\chi_{0, \varepsilon}\right\|_{V}^{4}+\left\|g_{e}\right\|_{L^{1}(0, T ; H)}^{2} .
$$

Remarking that $\chi_{\varepsilon}-\chi_{0, \varepsilon} \in \mathcal{V}$, by virtue of Poincaré inequality we obtain

$$
\begin{aligned}
\left\|\chi_{\varepsilon}(t)\right\|_{V}^{2} & \leq c\left(\left\|\nabla \chi_{\varepsilon}(t)\right\|_{H}^{2}+\left\|\chi_{0, \varepsilon}\right\|_{V}^{2}\right) \\
& \leq c\left(\left\|\vartheta_{0, \varepsilon}\right\|_{H}^{2}+\left\|\chi_{0, \varepsilon}\right\|_{V}^{4}+1+\left\|g_{\varepsilon}\right\|_{L^{1}(0, T ; H)}^{2}+\left\|\chi_{0, \varepsilon}\right\|_{V}^{2}\right) . \\
& \leq c\left(\left\|\vartheta_{0, \varepsilon}\right\|_{H}^{2}+\left\|\chi_{0, \varepsilon}\right\|_{V}^{4}+1+\left\|g_{\varepsilon}\right\|_{L^{1}(0, T ; H)}^{2}\right) \\
& =c B(\varepsilon)
\end{aligned}
$$

where $c>0$ is a constant. We point out that (2.28) yields $B(\varepsilon) \leq c_{2}, c_{2}>0$ being a constant.

Second set of a priori estimates. In order to deduce the second estimate we develop techniques which are similar to those employed in [7]. We first integrate (2.16) with respect to time to get

$$
\vartheta_{\varepsilon}+\ell_{\chi \varepsilon}-\vartheta_{0, \varepsilon}-\ell_{\chi_{0, \varepsilon}}+A\left(1 * k_{\varepsilon} * \vartheta_{\varepsilon}\right)=1 * g_{\varepsilon} \quad \text { in } V^{\prime}
$$

and multiply $(2.16)_{1}$ itself by $\varepsilon$ to get

$$
\varepsilon\left(\vartheta_{\varepsilon}+\ell_{\chi \epsilon}\right)^{\prime}+\varepsilon A\left(k_{\varepsilon} * \vartheta_{\epsilon}\right)=\varepsilon g_{\epsilon} \quad \text { in } V^{\prime}
$$

Adding the two equalities obtained this way and noting that (2.6) $-(2.7)$ yield $\varepsilon k_{\varepsilon}+1 *$ $k_{\varepsilon}=k_{0}+1 * \pi_{\varepsilon}$ we have

$$
\varepsilon\left(\vartheta_{\varepsilon}+\ell_{\chi_{\varepsilon}}\right)^{\prime}+\vartheta_{\epsilon}+\ell \chi_{\varepsilon}+A\left(\left(k_{0}+\Pi_{\varepsilon}\right) * \vartheta_{\varepsilon}\right)=F_{\varepsilon}
$$

where

$$
\begin{aligned}
& \Pi_{\varepsilon}=1 * \pi_{\varepsilon} \in W^{3,1}(0, T) \\
& F_{\varepsilon}=\varepsilon g_{\varepsilon}+\vartheta_{0, \varepsilon}+\ell \chi_{0, \varepsilon}+1 * g_{\varepsilon} \in L^{2}(0, T ; H) .
\end{aligned}
$$

We can rewrite (3.4) in terms of $w_{\varepsilon}$ as

$$
\varepsilon \partial_{\imath}^{2} w_{\varepsilon}+\partial_{\imath} w_{\varepsilon}+k_{0} A w_{\varepsilon}=F_{\varepsilon}-A\left(\pi_{\varepsilon} * w_{\varepsilon}\right)+A\left(\left(k_{0}+\Pi_{\varepsilon}\right) * \ell \chi_{\varepsilon}\right)
$$

Testing this by $\partial_{t} w_{\varepsilon}=\vartheta_{\varepsilon}+\ell \chi_{\varepsilon}$ and integrating with respect to $t$, we have

$$
\begin{aligned}
& \frac{\varepsilon}{2}\left\|\partial_{t} w_{\varepsilon}(t)\right\|_{H}^{2}+\iint_{Q_{t}}\left|\partial_{t} w_{\varepsilon}\right|^{2}+\frac{k_{0}}{2}\left\|\nabla w_{\varepsilon}(t)\right\|_{H}^{2} \\
& =\frac{\varepsilon}{2}\left\|\vartheta_{0, \varepsilon}+\ell \chi_{0, \varepsilon}\right\|_{H}^{2}+\int_{0}^{t}\left\langle F_{\varepsilon}(s), \partial_{t} w_{\varepsilon}(s)\right\rangle d s+\sum_{i=1}^{3} \mathcal{I}_{i}(t)
\end{aligned}
$$


where $Q_{t}=\Omega \times(0, t) \quad(t \in(0, T])$ and

$$
\begin{aligned}
& \mathcal{I}_{1}(t)=\int_{0}^{t}\left(\int_{\Omega} \nabla\left(k_{0} * \ell \chi_{\epsilon}\right)(s) \cdot \nabla\left(\partial_{t} w_{\epsilon}(s)\right)\right) d s \\
& \mathcal{I}_{2}(t)=\ell \iint_{Q_{t}}\left(\Pi_{\varepsilon} * \nabla \chi_{\epsilon}\right) \cdot \nabla\left(\partial_{t} w_{\varepsilon}\right) \\
& \mathcal{I}_{3}(t)=-\iint_{Q_{t}}\left(\pi_{\varepsilon} * \nabla w_{\varepsilon}\right) \cdot \nabla\left(\partial_{t} w_{\epsilon}\right) .
\end{aligned}
$$

We now estimate these quantities. Intcgrating by parts, we can treat $\mathcal{I}_{1}$ as

$$
\begin{aligned}
\mathcal{I}_{1}(t) & =\int_{\Omega}\left(k_{0} * \nabla\left(\ell \chi_{\epsilon}\right)\right)(t) \cdot \nabla w_{\varepsilon}(t)-\iint_{Q_{t}} \ell k_{0} \nabla \chi_{\varepsilon} \cdot \nabla w_{\varepsilon} \\
& \leq \sigma\left\|\nabla w_{\varepsilon}(t)\right\|_{H}^{2}+\frac{1}{4 \sigma}\left\|k_{0} * \nabla\left(\ell \chi_{\varepsilon}\right)\right\|_{L^{\infty}(0, t ; H)}^{2}-\iint_{Q_{t}} \ell k_{0} \nabla \chi_{\varepsilon} \cdot \nabla w_{\varepsilon} \\
. & \leq \sigma\left\|\nabla w_{\varepsilon}(t)\right\|_{H}^{2}+\frac{c}{4 \sigma} \ell^{2} k_{0}^{2} \iint_{Q_{t}}\left|\nabla \chi_{\varepsilon}\right|^{2}+\frac{k_{0} \ell}{2} \iint_{Q_{t}}\left|\nabla \chi_{\varepsilon}\right|^{2}+\frac{k_{0} \ell}{2} \iint_{Q_{t}}\left|\nabla w_{\varepsilon}\right|^{2}
\end{aligned}
$$

where the Young theorem and (2.35) have been employed.

To deal with $\mathcal{I}_{2}$, we use integration by parts and the Young inequality to obtain

$$
\begin{aligned}
\mathcal{I}_{2}(t)= & \ell \int_{\Omega}\left(\Pi_{\varepsilon} * \nabla \chi_{\epsilon}\right)(t) \cdot \nabla w_{\varepsilon}(t)-\ell \iint_{Q_{t}} \partial_{t}\left(\Pi_{\varepsilon} * \nabla \chi_{\varepsilon}\right) \cdot \nabla w_{\varepsilon} \\
= & \ell \int_{\Omega}\left(\Pi_{\varepsilon} * \nabla \chi_{\varepsilon}\right)(t) \cdot \nabla w_{\varepsilon}(t)-\ell \iint_{Q_{t}}\left(\pi_{\varepsilon} * \nabla \chi_{\varepsilon}\right) \cdot \nabla w_{\varepsilon} \\
\leq & \sigma\left\|\nabla w_{\varepsilon}(t)\right\|_{H}^{2}+\frac{1}{4 \sigma} \ell^{2}\left\|\Pi_{\varepsilon} * \nabla \chi_{\varepsilon}\right\|_{L^{\infty}(0, t ; H)}^{2} \\
& +\int_{0}^{t}\left\|\nabla w_{\varepsilon}(s)\right\|_{H}^{2} d s+\ell^{2} \int_{0}^{t}\left\|\left(\pi_{\varepsilon} * \nabla \chi_{\varepsilon}\right)(s)\right\|_{H}^{2} d s \\
\leq & \sigma\left\|\nabla w_{\varepsilon}(t)\right\|_{H}^{2}+\frac{1}{\sigma} \ell^{2} c\left\|\pi_{\varepsilon}\right\|_{L^{1}(0, T)}^{2} \iint_{Q_{t}}\left|\nabla \chi_{\varepsilon}\right|^{2} \\
& +\iint_{Q_{t}}\left|\nabla u_{\varepsilon}\right|^{2}+\ell^{2}\left\|\pi_{\varepsilon}\right\|_{L^{1}(0, T)}^{2} \iint_{Q_{t}}\left|\nabla \chi_{\varepsilon}\right|^{2} .
\end{aligned}
$$

As far as $\mathcal{I}_{3}$ is concerned, after integrating by parts and in view of (2.33) and (2.35) we have

$$
\begin{aligned}
\mathcal{I}_{3}(t) & =-\int_{\Omega}\left(\pi_{\varepsilon} * \nabla w_{\varepsilon}\right)(t) \cdot \nabla w_{\varepsilon}(t)+\iint_{Q_{t}}\left(\pi_{\varepsilon}(0) \nabla w_{\varepsilon}+\pi_{\varepsilon}^{\prime} * \nabla w_{\varepsilon}\right) \cdot \nabla w_{\varepsilon} \\
& \leq \sigma\left\|\nabla w_{\varepsilon}(t)\right\|_{H}^{2}+\frac{c}{\sigma}\left\|\pi_{\varepsilon}\right\|_{L^{2}(0, T)}^{2} \iint_{Q_{t}}\left|\nabla w_{\varepsilon}\right|^{2}+\left(\left|\pi_{\varepsilon}(0)\right|+\left\|\pi_{\varepsilon}^{\prime}\right\|_{L^{\prime}(0, T)}\right) \iint_{Q_{t}}\left|\nabla w_{\varepsilon}\right|^{2} .
\end{aligned}
$$

Now we collect all these estimates and add $\frac{k_{0}}{4}\left\|w_{\varepsilon}(t)\right\|_{H}^{2}$ to both sides of (3.7). Remarking that

$$
\frac{k_{0}}{4}\left\|w_{\varepsilon}(t)\right\|_{H}^{2} \leq c \int_{0}^{t}\left\|w_{\epsilon}(s)\right\|_{H}\left\|\partial_{t} w_{\epsilon}(s)\right\|_{H} d s \leq \sigma \iint_{Q_{t}}\left|\partial_{t} w_{\varepsilon}\right|^{2}+\frac{c}{\sigma} \iint_{Q_{t}}\left|w_{\varepsilon}\right|^{2}
$$


we infer that

$$
\begin{aligned}
& \frac{\varepsilon}{2}\left\|\partial_{t} w_{\varepsilon}(t)\right\|_{H}^{2}+\iint_{Q_{t}}\left|\partial_{t} w_{\varepsilon}\right|^{2}+\frac{k_{0}}{2}\left\|\nabla w_{\varepsilon}(t)\right\|_{H}^{2}+\frac{k_{0}}{4}\left\|w_{\varepsilon}(t)\right\|_{H}^{2} \\
& \leq \varepsilon\left\|\vartheta_{0, \varepsilon}\right\|_{H}^{2}+\varepsilon \ell^{2}\left\|\chi_{0, \varepsilon}\right\|_{H}^{2}+\sigma\left\|\nabla w_{\varepsilon}(t)\right\|_{H}^{2}+\frac{1}{\sigma} c k_{0}^{2} \ell^{2} \iint_{Q_{t}}\left|\nabla \chi_{\varepsilon}\right|^{2} \\
& \quad+\frac{k_{0} \ell}{2} \iint_{Q_{t}}\left|\nabla \chi_{\varepsilon}\right|^{2}+\frac{k_{0} \ell}{2} \iint_{Q_{t}}\left|\nabla w_{\varepsilon}\right|^{2}+\frac{c}{\sigma} \int_{0}^{t}\left\|F_{\varepsilon}(s)\right\|_{H}^{2} d s \\
& \quad+\sigma \int_{0}^{t}\left\|\partial_{t} w_{\varepsilon}(s)\right\|_{H}^{2} d s+\sigma\left\|\nabla w_{\varepsilon}(t)\right\|_{H}^{2}+\frac{1}{\sigma} \ell^{2} c\left\|\pi_{\varepsilon}\right\|_{L^{1}(0, T)}^{2} \iint_{Q_{t}}\left|\nabla \chi_{\varepsilon}\right|^{2} \\
& \quad+\iint_{Q_{t}}\left|\nabla w_{\varepsilon}\right|^{2}+\ell^{2}\left\|\pi_{\varepsilon}\right\|_{L^{1}(0, T)}^{2} \iint_{Q_{t}}\left|\nabla \chi_{\varepsilon}\right|^{2}+\sigma\left\|\nabla w_{\varepsilon}(t)\right\|_{H}^{2} \\
& \quad+c\left(\frac{1}{\sigma}\left\|\pi_{\varepsilon}\right\|_{L^{2}(0, T)}^{2}+\left|\pi_{\varepsilon}(0)\right|+\left\|\pi_{\varepsilon}^{\prime}\right\|_{L^{1}(0, T)}\right) \iint_{Q_{t}}\left|\nabla w_{\varepsilon}\right|^{2} \\
& \quad+\sigma \iint_{Q_{t}}\left|\partial_{t} w_{\varepsilon}\right|^{2}+\frac{c}{\sigma} \iint_{Q_{t}}\left|w_{\epsilon}\right|^{2} .
\end{aligned}
$$

If we choose $\sigma=\min \left\{\frac{1}{4}, \frac{k_{0}}{12}\right\}$ and recall (3.2) - (3.3) we obtain

$$
\begin{aligned}
\frac{\varepsilon}{2}\left\|\partial_{t} w_{\varepsilon}(t)\right\|_{H}^{2}+\frac{1}{2} \iint_{Q_{t}}\left|\partial_{t} w_{\varepsilon}\right|^{2}+\frac{k_{0}}{4}\left\|w_{\varepsilon}(t)\right\|_{V}^{2} \\
\leq \varepsilon\left\|\vartheta_{0, \varepsilon}\right\|_{H}^{2}+\varepsilon \ell^{2}\left\|\chi_{0, \varepsilon}\right\|_{H}^{2}+\left(c+c\left\|\pi_{\varepsilon}\right\|_{L^{1}(0, T)}^{2}\right) \iint_{Q_{\varepsilon}}\left|\nabla \chi_{\varepsilon}\right|^{2} \\
\quad+\left(c+c\left\|\pi_{\varepsilon}\right\|_{L^{2}(0, T)}^{2}+\left|\pi_{\varepsilon}(0)\right|+\left\|\pi_{\varepsilon}^{\prime}\right\|_{L^{1}(0, T)}\right) \iint_{Q_{t}}\left|\nabla w_{\varepsilon}\right|^{2} \\
\quad+c \int_{0}^{l}\left\|F_{\varepsilon}(s)\right\|_{H}^{2} d s+c \iint_{Q_{t}}\left|w_{\varepsilon}\right|^{2} \\
\leq \varepsilon\left\|\vartheta_{0, \varepsilon}\right\|_{H}^{2}+\varepsilon \ell^{2}\left\|\chi_{0, \varepsilon}\right\|_{H}^{2}+c B(\varepsilon)\left(1+\left\|\pi_{\varepsilon}\right\|_{L^{2}(0, T)}^{2}\right) \\
\quad+c\left\|F_{\varepsilon}\right\|_{L^{2}(0, T ; H)}^{2}+G(\varepsilon) \int_{0}^{t}\left\|w_{\varepsilon}(s)\right\|_{V}^{2} d s
\end{aligned}
$$

where we have set

$$
G(\varepsilon)=c+c\left\|\pi_{\varepsilon}\right\|_{L^{2}(0, T)}^{2}+\left|\pi_{\varepsilon}(0)\right|+\left\|\pi_{\varepsilon}^{\prime}\right\|_{L^{1}(0, T)}
$$

for some constant $c>0$. Applying the Gronwall lemma we find the estimate

$$
\begin{gathered}
\varepsilon\left\|\partial_{t} w_{\varepsilon}(t)\right\|_{H}^{2}+\iint_{Q_{t}}\left|\partial_{t} w_{e}\right|^{2}+\left\|w_{\epsilon}(t)\right\|_{V}^{2} \\
\leq c\left(\varepsilon\left\|\vartheta_{0, \epsilon}\right\|_{H}^{2}+\varepsilon \ell^{2}\left\|\chi_{0, \epsilon}\right\|_{H}^{2}+B(\varepsilon)\left(1+\left\|\pi_{\varepsilon}\right\|_{L^{1}(0, T)}^{2}\right)+\left\|F_{\varepsilon}\right\|_{L^{2}\left(0, T^{\prime} ; H\right)}^{2}\right) e^{G(\varepsilon) T} .
\end{gathered}
$$

Since (2.26) implies

$$
\left\|\pi_{\varepsilon}\right\|_{W^{1,1}(0, T)} \leq B_{1}
$$


for some constant $B_{1}>0$, we have $G(\varepsilon) \leq B_{2}$ so that, recalling also (2.28), we infer

$$
\varepsilon\left\|\partial_{t} w_{\varepsilon}(t)\right\|_{H}^{2}+\iint_{Q_{t}}\left|\partial_{t} w_{\varepsilon}\right|^{2}+\left\|w_{\varepsilon}(t)\right\|_{V}^{2} \leq B_{3}
$$

for some constant $B_{3}>0$ depending on $\Omega, k_{0}, \ell, T$. Besides, the estimate

$$
\begin{aligned}
\left\|\left(1 * \vartheta_{\varepsilon}\right)(t)\right\|_{V} & \leq\left\|\left(1 *\left(\vartheta_{\epsilon}+\ell_{\chi_{\varepsilon}}\right)\right)(t)\right\|_{V}+\ell\left\|\left(1 * \chi_{\varepsilon}\right)(t)\right\|_{V} \\
& \leq\left\|w_{\varepsilon}(t)\right\|_{V}+c\left\|_{\varepsilon}\right\|_{L^{2}(0, T ; V)} \\
& \leq B_{3}^{\frac{1}{2}}+c B(\varepsilon)^{\frac{1}{2}} \\
& \leq B_{4}
\end{aligned}
$$

holds for some constant $B_{4}^{-}>0$ depending on $\Omega, k_{0}, \ell, T$.

Third set of a priori estimates. By $(2.16)_{2}$ tested by $v=\xi_{\varepsilon}(t)$ and integrated over $(0, T)$, we have

$$
\left\|\nabla \xi_{\varepsilon}\right\|_{L^{2}(0, T ; H)}^{2} \leq\left\|\xi_{\varepsilon}\right\|_{L^{2}(0, T ; V)}\left\|\partial_{t} \chi_{\varepsilon}\right\|_{L^{2}\left(0, T ; V^{\prime}\right)} \leq c\left\|\xi_{\varepsilon}\right\|_{L^{2}(0, T ; V)}
$$

where (3.1) is used to get the second inequality. We put

$$
M_{\varepsilon}(t)=\frac{1}{|\Omega|} \int_{\Omega} \xi_{\varepsilon}(t) d x .
$$

The Poincaré inequality, (3.11) and (2.35) next yield

$$
\begin{aligned}
\left\|\xi_{\varepsilon}\right\|_{L^{2}(0, T ; V)}^{2} & \leq c\left\|\xi_{\varepsilon}-M_{\varepsilon}\right\|_{L^{2}(0, T ; V)}^{2}+c\left|M_{\varepsilon}\right|_{L^{2}(0, T)}^{2} \\
& \leq c\left\|\nabla \xi_{\varepsilon}\right\|_{L^{2}(0, T ; H)}^{2}+c\left|M_{e}\right|_{L^{2}(0, T)}^{2} \\
& \leq c\left\|\xi_{\varepsilon}\right\|_{L^{2}(0, T ; V)}+c\left|M_{\epsilon}\right|_{L^{2}(0, T)}^{2} \\
& \leq \frac{1}{2}\left\|\xi_{\varepsilon}\right\|_{L^{2}(0, T ; V)}^{2}+c+c\left|M_{\varepsilon}\right|_{L^{2}(0, T)}^{2}
\end{aligned}
$$

Thus

$$
\left\|\xi_{\varepsilon}\right\|_{L^{2}(0, T ; V)}^{2} \leq c^{\prime}+c\left|M_{\varepsilon}\right|_{L^{2}(0, T)}^{2}
$$

and it easily follows from (3.1) and $(2.16)_{3}$ that $M_{\varepsilon}$ is bounded indipendently of $\varepsilon$ in $L^{\infty}(0, T)$, hence

$$
\int_{0}^{T}\left\|\xi_{\varepsilon}(t)\right\|_{v}^{2} d t \leq B_{5}
$$

for some constant $B_{5}>0$ depending on $\Omega, k_{0}, \ell, T$. The continuous embedding $H^{1}(\Omega) \subset$ $L^{6}(\Omega)$ yields

$$
\left\|\chi_{\varepsilon}^{3}(t)\right\|_{H}^{2}=\left\|\chi_{e}(t)\right\|_{L^{B}(\Omega)}^{6} \leq c\left\|\chi_{e}(t)\right\|_{V}^{6} \leq c B(\varepsilon)^{3}
$$

and therefore

$$
\left\|\chi_{\varepsilon}^{3}(t)\right\|_{H} \leq B_{6}
$$


for some constant $B_{6}>0$.

Weak convergence. The previous estimates yield

$$
\left.\begin{array}{r}
\left\|\vartheta_{\varepsilon}\right\|_{L^{\infty}(0, T ; H)} \\
\left\|1 * \vartheta_{\varepsilon}\right\|_{L^{\infty}(0, T ; V)} \\
\left\|\chi_{\varepsilon}\right\|_{L^{\infty}(0, T ; V) \cap H^{\prime}\left(0, T ; V^{\prime}\right) \cap L^{\infty}\left(0, T ; L^{4}(\Omega)\right)} \\
\left\|\xi_{\varepsilon}\right\|_{L^{2}(0, T ; V)} \\
\left\|\chi_{\varepsilon}^{3}\right\|_{L^{\infty}(0, T ; H)}
\end{array}\right\} \leq C\left(\Omega, k_{0}, \ell, T\right) .
$$

Well-known weak or weak star compactness results ensure the existence of

$$
\begin{aligned}
& \vartheta \in L^{\infty}(0, T ; H) \\
& \psi \in L^{\infty}(0, T ; V) \\
& \chi \in L^{\infty}(0, T ; V) \cap H^{1}\left(0, T ; V^{\prime}\right) \cap L^{\infty}\left(0, T ; L^{4}(\Omega)\right) \\
& \xi \in L^{2}(0, T ; V) \\
& \varphi \in L^{\infty}(0, T ; H)
\end{aligned}
$$

such that, at least for a subsequence of $\dot{\varepsilon} \downarrow 0$, the convergences

$$
\begin{array}{rll}
\vartheta_{\varepsilon} \stackrel{\star}{\rightarrow} \vartheta & \text { in } & L^{\infty}(0, T ; H) \\
1 * \vartheta_{\varepsilon} \stackrel{\star}{\psi} \psi & \text { in } & L^{\infty}(0, T ; V) \\
\chi_{\varepsilon} \stackrel{\star}{\rightarrow} \chi & \text { in } & L^{\infty}(0, T ; V) \cap H^{1}\left(0, T ; V^{\prime}\right) \cap L^{\infty}\left(0, T ; L^{4}(\Omega)\right) \\
\xi_{\varepsilon} \rightarrow \xi & \text { in } & L^{2}(0, T ; V) \\
\chi_{\varepsilon}^{3} \stackrel{*}{ } \varphi & \text { in } & L^{\infty}(0, T ; H)
\end{array}
$$

hold. It is casy to show $\psi=1 * \vartheta$, so that

$$
1 * \vartheta_{e} \stackrel{\star}{\rightarrow} 1 * \vartheta \quad \text { in } L^{\infty}(0, T ; V)
$$

In order to prove $\varphi=\chi^{3}$, we would like $\chi_{\varepsilon}$ to converge to $\chi$ in a quite strong sense. Using [14: p. 89/Corollary 8], we obtain that $\left\{\chi_{\varepsilon}\right\}$ is relatively compact in $C^{0}([0, T] ; H)$ so that, possibly for a subsequence of $\varepsilon \downarrow 0$,

$$
\chi_{\varepsilon} \rightarrow \chi \quad \text { in } C^{0}([0, T] ; H) .
$$

Then $\chi_{\varepsilon} \rightarrow \chi$ in $L^{2}\left(Q_{T}\right)$ and, possibly for a subsequence, a.e. in $Q_{T}$, and this implies $\chi_{\varepsilon}^{3} \rightarrow \chi^{3}$ a.c. in $Q_{T}$. Thus $\chi^{3}=\varphi$ a.e. in $Q_{T}$ and we get

$$
\chi_{\varepsilon}^{3} \stackrel{*}{*} \chi^{3} \quad \text { in } L^{\infty}(0, T ; H) .
$$

Passage to limit. Owing to (2.24), (2.16) 4 and (3.17), we have

$$
\left.\begin{array}{rl}
\chi_{\varepsilon}(0)=\chi_{0, \varepsilon} & \rightarrow \chi_{0} \\
\chi_{\varepsilon}(0) & \rightarrow \chi(0)
\end{array}\right\} \quad \text { in } H
$$


and consequently $\chi(0)=\chi_{0}$. Now we let $\varepsilon \downarrow 0$ in $(2.16)_{3}$. Then $(3.15)_{3},(3.18),(3.15)_{1}$, (3.15) ${ }_{4}$ and the fact that $A \in \mathcal{L}\left(V, V^{\prime}\right)$ allow us to pass to limit in $L^{2}\left(0, t ; V^{\prime}\right)$ in a weak sense, thus obtaining $(2.19)_{3}$. Thanks to $(3.15)_{3}$ and $(3.15)_{4}$ we can pass to limit in $(2.16)_{2}$ with respect to the wcak topology of $L^{2}\left(0, T ; V^{\prime}\right)$ and we get $(2.19)_{2}$.

We now want to pass to the limit in (3.4). First of all we can rewrite (3.4) as

$$
\varepsilon \partial_{t}^{2} w_{\varepsilon}+\partial_{t} w_{\varepsilon}+k_{0} A\left(1 * \vartheta_{\varepsilon}\right)+A\left(1 * \pi_{\varepsilon} * \vartheta_{\varepsilon}\right)=F_{\varepsilon}
$$

Next, by (3.5) we have

$$
\begin{aligned}
&\left\|\varepsilon \partial_{t}^{2} w_{\varepsilon}\right\|_{L^{2}\left(0, T_{;} V^{\prime}\right)}^{2} \\
& \leq c\left(\left\|\partial_{t} w_{\varepsilon}\right\|_{L^{2}\left(0, T ; V^{\prime}\right)}^{2}+k_{0}^{2}\|A\|_{L\left(V, V^{\prime}\right)}^{2}\left\|1 * \vartheta_{\varepsilon}\right\|_{L^{2}(0, T ; V)}^{2}\right. \\
&+\varepsilon^{2}\left\|g_{\varepsilon}\right\|_{L^{2}\left(0, T ; V^{\prime}\right)}^{2}+\left\|g_{\varepsilon}\right\|_{L^{2}\left(0, T ; V^{\prime}\right)}^{2}+\left\|\vartheta_{0, \varepsilon}\right\|_{V^{\prime}}^{2}+\left\|\chi_{0, \varepsilon}\right\|_{V^{\prime}}^{2} \\
&\left.+\|A\|_{\mathcal{L}\left(V, V^{\prime}\right)}^{2}\left\|1 * \pi_{\varepsilon} * \vartheta_{\varepsilon}\right\|_{L^{2}(0, T ; V)}^{2}\right) \\
& \leq c\left(\iint_{Q_{t}}\left|\partial_{t} w_{\varepsilon}\right|^{2}+k_{0}^{2}\|A\|_{\mathcal{L}\left(V, V^{\prime}\right)}^{2}\left\|1 * \vartheta_{\varepsilon}\right\|_{L^{\infty}(0, T ; V)}^{2} \cdot\right. \\
&+\left\|g_{\varepsilon}\right\|_{L^{2}(0, T ; H)}^{2}+\left\|g_{\varepsilon}\right\|_{L^{2}(0, T ; H)}^{2}+\left\|\vartheta_{0, \varepsilon}\right\|_{H}^{2}+\left\|\chi_{0, \varepsilon}\right\|_{V}^{2} \\
&\left.+\|A\|_{\mathcal{L}\left(V, V^{\prime}\right)}^{2}\left\|\pi_{\varepsilon}\right\|_{L^{1}(0, T)}^{2}\left\|1 * \vartheta_{\varepsilon}\right\|_{L^{\infty}(0, T ; V)}^{2}\right) .
\end{aligned}
$$

Therefore, from (3.9), (2.28), (3.10) and (3.8) we get

$$
\left\|\varepsilon \partial_{t}^{2} w_{\varepsilon}\right\|_{L^{2}\left(0, T ; V^{\prime}\right)} \leq B_{7}
$$

for some constant $B_{7}>0$. Then the previous estimate and (3.9) (which ensures that $\varepsilon \partial_{t} w_{\varepsilon} \rightarrow 0$ in the sense of distributions) allow us to conclude

$$
\varepsilon \partial_{i}^{2} w_{\varepsilon} \rightarrow 0 \quad \text { in } L^{2}\left(0, T ; V^{\prime}\right)
$$

We can note that $(3.15)_{1}$ and $(3.15)_{3}$ imply $\partial_{t} w_{\varepsilon} \rightarrow \vartheta+\ell \chi$ in $L^{2}\left(0, T ; V^{\prime}\right)$ and (3.16). yields $A\left(1 * \vartheta_{\varepsilon}\right) \rightarrow A(1 * \vartheta)$ in $L^{2}\left(0, T ; V^{\prime}\right)$. By $(2.24)$ and $(2.25)$ we have

$$
\left.\begin{array}{rlrl}
\varepsilon g_{\varepsilon} & \rightarrow 0 & & \text { in } L^{2}\left(0, T ; V^{\prime}\right) \\
1 * g_{\varepsilon} & \rightarrow 1 * g & & \text { in } L^{2}\left(0, T ; V^{\prime}\right) \\
\vartheta_{0, \varepsilon} & \rightarrow \vartheta_{0} & & \text { in } V^{\prime}
\end{array}\right\} .
$$

Moreover, recalling the Young theorem, we get

$$
\begin{aligned}
\left\|A\left(1 * \pi_{\varepsilon} * \vartheta_{\varepsilon}\right)\right\|_{L^{2}\left(0, T ; V^{\prime}\right)} & \leq\|A\|_{\mathcal{L}\left(V, V^{\prime}\right)}\left\|1 * \pi_{\epsilon} * \vartheta_{\epsilon}\right\|_{L^{2}(0, T ; V)} \\
& \leq\|A\|_{\mathcal{L}\left(V, V^{\prime}\right)}\left\|1 * \vartheta_{\epsilon}\right\|_{L^{2}(0, T ; V)}\left\|\pi_{\varepsilon}\right\|_{L^{1}(0, T)} \\
& \leq c\|A\|_{\mathcal{L}\left(V, V^{\prime}\right)} B_{4}\left\|\pi_{\varepsilon}\right\|_{W^{1,1}(0, T)}
\end{aligned}
$$

and $\left\|\pi_{\varepsilon}\right\|_{W^{1,1}(0, T)} \rightarrow 0$ thanks to $(2.26)$. Thus $A\left(1 * \pi_{\varepsilon} * \vartheta_{\varepsilon}\right) \rightarrow 0$ in $L^{2}\left(0, T ; V^{\prime}\right)$. Passing now to the limit in (3.19) we obtain

$$
\vartheta+\ell \chi+k_{0} A(1 * \vartheta)=1 * g+\vartheta_{0}+\ell_{\chi_{0}}
$$


in $L^{2}\left(0, T ; V^{\prime}\right)$ and consequently in $V^{\prime}$, a.e. in $(0, T)$.

Introducing now the operator $\tilde{A} \in \mathcal{L}\left(H, W^{\prime}\right)$ by

$$
\langle(\tilde{A} v, z\rangle\rangle=-\int_{\Omega} v \Delta z \quad(z \in W, v \in H)
$$

we can observe that $\widetilde{A}$ is an extension of $A$. Since $1 * \vartheta \in H^{1}(0, T ; H)$, we have $\tilde{A}(1 * \vartheta)=$ $A(1 * \vartheta) \in H^{1}\left(0, T ; W^{\prime}\right)$. Since $\chi$ and $1 * g$ are in $H^{1}\left(0, T ; W^{\prime}\right)$ too, by $(3.20)$ we get $\vartheta \in H^{1}\left(0, T ; W^{\prime}\right)$. Differentiating $(3.20)$ with respect to $t$, we find $\partial_{t}(\vartheta+\ell \chi)+k_{0} A \vartheta=g$ in $L^{2}\left(0, T ; W^{\prime}\right)$ and thus in $W^{\prime}$, a.c. in $(0, T)$. Finally, $(3.20)$ in $t=0$ gives $\vartheta(0)=\vartheta_{0}$ in account of the fact that $\chi(0)=\chi_{0}$.

It remains to verify the strong convergence in $(2.29)_{1}$ and $(2.29)_{2}$, and the weak convergences of $\vartheta_{\varepsilon}$ to $\vartheta$ in $H^{1}\left(0, T ; W^{\prime}\right)$ and of $\chi_{\varepsilon}$ to $\chi$ in $L^{2}(0, T ; W)$.

First we prove $\left\{\chi_{\varepsilon}\right\}$ is bounded in $L^{2}(0, T ; W)$. By $(2.16)_{3}$ and $(3.14)_{1,3-5}$ we have

$$
\begin{aligned}
& \left\|A \chi_{\varepsilon}\right\|_{L^{2}(0, T ; H)} \\
& \quad \leq\left\|\chi_{\varepsilon}\right\|_{L^{2}(0, T ; H)}+\left\|\chi_{\varepsilon}^{3}\right\|_{L^{2}(0, T ; H)}+\ell\left\|\vartheta_{\varepsilon}\right\|_{L^{2}(0, T ; H)}+\left\|\xi_{\varepsilon}\right\|_{L^{2}(0, T ; H)} \\
& \quad \leq c\left(\Omega, k_{o}, \ell, T\right) . .
\end{aligned}
$$

Hence, thanks to well-known regularity results on elliptic equations, we conclude

$$
\left\|\chi_{\varepsilon}\right\|_{L^{2}(0, T ; W)} \leq c\left(\Omega, k_{0}, \ell, T\right)
$$

Then, possibly taking a subsequence of $\varepsilon \downarrow 0$, there exists $u \in L^{2}(0, T ; W)$ such that $\chi_{\varepsilon}-u$ in $L^{2}(0, T ; W)$. From $(3.15)_{3}$ it is clear that $u=\chi$, so

$$
\chi_{\epsilon} \stackrel{\star}{=} \chi \quad \text { in } L^{\infty}(0, T ; V) \cap H^{1}\left(0, T ; V^{\prime}\right) \cap L^{\infty}\left(0, T ; L^{4}(\Omega)\right) \cap L^{2}(0, T ; W) .
$$

Being $\left\{\chi_{\epsilon}\right\}$ bounded in $L^{2}(0, T ; W)$ and $\left\{\partial_{t} \chi_{\epsilon}\right\}$ bounded in $L^{2}\left(0, T ; V^{\prime}\right)$, by virtue of [14: p. 89/Corollary 8] we conclude (possibly for a subsequence) $\chi_{\varepsilon} \rightarrow \chi$ in $L^{2}(0, T ; V)$. Being, by $(3.14)_{2},\left\{1 * \vartheta_{\varepsilon}\right\}$ bounded in $L^{\infty}(0, T ; V)$ and, by $(3.14)_{1},\left\{\vartheta_{\varepsilon}\right\}$ bounded in

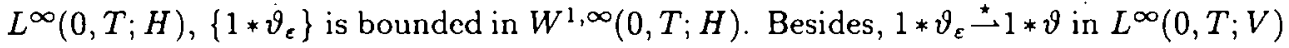
so that we can apply the Ascoli theorem and conclude

$$
1 * \vartheta_{\varepsilon} \rightarrow 1 * \vartheta \quad \text { in } C^{0}([0, T] ; H)
$$

Since $k_{\varepsilon} * \vartheta_{\varepsilon}$ belongs to $C^{0}([0, T] ; H)$ (and thus to $\left.L^{2}(0, T ; H)\right)$ as $k_{\varepsilon} \in C^{0}([0, T])$ and $\vartheta_{\varepsilon} \in L^{\infty}(0, T ; H)$, we have $A\left(k_{\varepsilon} * \vartheta_{\varepsilon}\right) \in L^{2}\left(0, T ; W^{\prime}\right)$. Thanks to $(2.16)_{1}$, we find $\partial_{\imath} \vartheta_{\varepsilon} \in L^{2}\left(0, T ; W^{\prime}\right)$, and $(3.14)_{1}$ yields

$$
\begin{aligned}
\left\|A\left(k_{\varepsilon} * \vartheta_{\varepsilon}\right)\right\|_{L^{2}\left(0, T ; W^{\prime}\right)} & \leq\|\tilde{A}\|_{\mathcal{L}\left(H, W^{\prime}\right)}\left\|k_{\varepsilon} * \vartheta_{\varepsilon}\right\|_{L^{2}(0, T ; H)} \\
& \leq c\|\tilde{A}\|_{\mathcal{L}\left(H, W^{\prime}\right)}\left\|\vartheta_{\varepsilon}\right\|_{L^{2}(0, T ; H)} \\
& \leq c
\end{aligned}
$$

for some constant $c>0$ depending on $\Omega, k_{0}, \ell, T$, where $0 \leq \int_{0}^{T} k_{\varepsilon}(t) d t \leq c$ has been used. Since by $(2.28)\left\{g_{\varepsilon}\right\}$ is bounded in $L^{2}\left(0, T ; W^{\prime}\right)$ and by $(3.14)_{3}\left\|\partial_{t} \chi_{\varepsilon}\right\|_{L^{2}\left(0, T ; W^{\prime}\right)}$ is 
bounded we get $\left\|\partial_{t} \vartheta_{\epsilon}\right\|_{L^{2}\left(0, T ; W^{\prime}\right)} \leq c$. Thus $\partial_{t} \vartheta_{\varepsilon} \rightarrow \partial_{t} \vartheta$ in $L^{2}\left(0, T ; W^{\prime}\right)$ and so $\vartheta_{\varepsilon} \rightarrow \vartheta$ in $H^{1}\left(0, T ; W^{\prime}\right)$. Thanks to [14: p. $89 /$ Corollary 8 ] again, $\left\{\vartheta_{\varepsilon}\right\}$ is relatively compact in $L^{2}\left(0, T ; V^{\prime}\right)$. Hence, possibly for a subsequence,

$$
\vartheta_{\varepsilon} \rightarrow \vartheta \quad \text { in } L^{2}\left(0, T ; V^{\prime}\right)
$$

Therefore (3.22) and (3.23) yield $1 * \vartheta_{\varepsilon} \rightarrow 1 * \vartheta$ in $H^{1}\left(0, T ; V^{\prime}\right)$. Finally, let us remark that the uniqueness of the solution to the limit problem implies that the convergences stated above hold for the entire sequence

\section{Proof of Theorem 2.2}

First of all, we prove an estimate for

$$
\varepsilon^{\frac{1}{2}}\left\|\partial_{t}^{2} w_{\varepsilon}\right\|_{L^{2}(0, T ; H)}
$$

following the scheme of [7]. We differentiate (3.6) with respect to $t$ and we would like testing it by $\varepsilon \partial_{t}^{2} w_{\varepsilon}$. Since this is not an admissible test function, we should approximate it by admissible test functions, e.g., as in [6: Appendix]. However, we prefer to proceed formally and use $\varepsilon \partial_{t}^{2} w_{\varepsilon}$ directly, since we are essentially allowed to do it. We integrate over $(0, t)$ and, recovering the initial value for $\partial_{t}^{2} w_{\epsilon}$ from $(2.18)_{1}$, we obtain

$$
\begin{aligned}
& \frac{\varepsilon^{2}}{2}\left\|\partial_{t}^{2} w_{\varepsilon}(t)\right\|_{H}^{2}+\varepsilon \iint_{Q_{t}}\left|\partial_{t}^{2} w_{\varepsilon}\right|^{2}+\frac{k_{0} \varepsilon}{2}\left\|\nabla \partial_{t} w_{\varepsilon}(t)\right\|_{H}^{2} \\
& =\frac{\varepsilon^{2}}{2}\left\|g_{\varepsilon}(0)\right\|_{H}^{2}+\frac{k_{0} \varepsilon}{2}\left\|\nabla\left(\vartheta_{0, \varepsilon}+\ell \chi_{0, \varepsilon}\right)\right\|_{H}^{2}+\sum_{i=1}^{3} \mathcal{I}_{i}(t)
\end{aligned}
$$

where

$$
\begin{aligned}
& \mathcal{I}_{1}(t)=\varepsilon \int_{0}^{t}\left\langle\partial_{t} F_{\varepsilon}(s), \partial_{t}^{2} w_{\epsilon}(s)\right\rangle d s \\
& I_{2}(t)=-\varepsilon \int_{0}^{t}\left\langle A \partial_{t}\left(\pi_{\epsilon} * w_{\varepsilon}\right)(s), \partial_{t}^{2} w_{\varepsilon}(s)\right\rangle d s \\
& I_{3}(t)=\varepsilon \int_{0}^{t}\left\langle A \partial_{t}\left(\left(k_{0}+\Pi_{\epsilon}\right) * \ell_{\chi_{\epsilon}}\right)(s), \partial_{t}^{2} w_{\epsilon}(s)\right\rangle d s
\end{aligned}
$$

Now we estimate these quantities. Since (2.30) and (3.5) imply

$$
F_{\varepsilon} \in H^{1}(0, T ; H)+W^{2,1}\left(0, T ; V^{\prime}\right)
$$

we split

$$
F_{\varepsilon}=F_{\varepsilon, 1}+F_{\varepsilon, 2} \quad \text { with }\left\{\begin{array}{l}
F_{\varepsilon, 1} \in H^{1}(0, T ; H) \\
F_{\varepsilon, 2} \in W^{2,1}\left(0, T ; V^{\prime}\right)
\end{array}\right.
$$


In $\mathcal{I}_{1}(t)$ we integrate by parts the second term, and owing to $(2.18)_{4}$ we have

$$
\begin{aligned}
\mathcal{I}_{1}(t) \leq & \sigma \varepsilon \iint_{Q_{t}}\left|\partial_{t}^{2} w_{\varepsilon}\right|^{2}+c_{\sigma} \varepsilon \iint_{Q_{t}}\left|\partial_{t} F_{\varepsilon, 1}\right|^{2}+\sigma \varepsilon\left\|\partial_{t} w_{\varepsilon}(t)\right\|_{V}^{2} \\
& +c_{\sigma} \varepsilon\left\|\partial_{t} F_{\varepsilon, 2}(t)\right\|_{V^{\prime}}^{2}+\varepsilon\left\|\vartheta_{0, \varepsilon}+\ell \chi_{0, \varepsilon}\right\|_{V}^{2}+\varepsilon\left\|\partial_{t} F_{\varepsilon, 2}(0)\right\|_{V^{\prime}}^{2} \\
& +\int_{0}^{t} \varepsilon^{\frac{1}{2}}\left\|\partial_{t}^{2} F_{\varepsilon, 2}(s)\right\|_{V^{\prime}} \varepsilon^{\frac{1}{2}}\left\|\partial_{t} w_{\varepsilon}(s)\right\|_{V} d s
\end{aligned}
$$

In order to deal with $\mathcal{I}_{2}$, we use (2.33), (2.1) and integrate by parts. Owing to (3.8) and (3.9), we obtain

$$
\begin{aligned}
\mathcal{I}_{2}(t)= & -\varepsilon \iint_{Q_{t}} \nabla\left(\pi_{\varepsilon}(0) w_{\epsilon}+\pi_{\varepsilon}^{\prime} * w_{\varepsilon}\right) \cdot \nabla \partial_{t}^{2} w_{\varepsilon} \\
= & -\varepsilon \iint_{\Omega} \nabla\left(\pi_{\varepsilon}(0) w_{\epsilon}+\pi_{\varepsilon}^{\prime} * w_{\varepsilon}\right)(t) \cdot \nabla \partial_{t} w_{\varepsilon}(t) \\
& +\varepsilon \iint_{Q_{t}} \nabla\left(\pi_{\varepsilon}(0) \partial_{t} w_{\varepsilon}+\pi_{\varepsilon}^{\prime} * \partial_{t} w_{\varepsilon}\right) \cdot \nabla \partial_{t} w_{\varepsilon} \\
\leq & \sigma \varepsilon\left\|\nabla \partial_{t} w_{\varepsilon}(t)\right\|_{H}^{2}+\varepsilon c_{\sigma} B_{1}^{2}\left\|\nabla w_{\varepsilon}(t)\right\|_{H}^{2} \\
& +c_{\sigma} \varepsilon\left\|\left(\pi_{\varepsilon}^{\prime} * \nabla w_{\epsilon}\right)(t)\right\|_{H}^{2}+\varepsilon B_{1} c \iint_{Q_{t}} \cdot\left|\nabla \partial_{t} w_{\varepsilon}\right|^{2} \\
& \quad \sigma \varepsilon\left\|\nabla \partial_{t} w_{\varepsilon}(t)\right\|_{H}^{2}+\varepsilon c_{\sigma, B_{1}}\left(B_{3}+\iiint_{Q_{t}}\left|\nabla \partial_{t} w_{\varepsilon}\right|^{2}\right)
\end{aligned}
$$

At last, we consider $\mathcal{I}_{3}$ and, in view of $(2.34),(3.21)$ and (3.8), we get

$$
\begin{aligned}
\mathcal{I}_{3}(t) \leq & \varepsilon \int_{0}^{t}\left\|\Delta\left(k_{0} \ell \chi_{\varepsilon}+\pi_{\varepsilon} * \ell \chi_{\varepsilon}\right)(s)\right\|_{H}\left\|\partial_{t}^{2} w_{\varepsilon}(s)\right\|_{H} d s \\
\leq & c \varepsilon \int_{0}^{t}\left\|\Delta \chi_{\varepsilon}(s)\right\|_{H}\left\|\partial_{t}^{2} w_{\varepsilon}(s)\right\|_{H} d s \\
& +\ell \varepsilon\left\|\pi_{\varepsilon}\right\|_{L^{\infty}(0, T)}\left\|\chi_{\varepsilon}\right\|_{L^{1}(0, T ; W)}\left\|\partial_{t}^{2} w_{\varepsilon}\right\|_{L^{1}(0, T ; H)} \\
\leq & c \int_{0}^{t}\left(\left\|\chi_{\varepsilon}(s)\right\|_{W}+c\right) \varepsilon\left\|\partial_{t}^{2} w_{\varepsilon}(s)\right\|_{H} d s .
\end{aligned}
$$

Then we add $\frac{k_{0} \varepsilon}{2}\left\|\partial_{t} w_{\varepsilon}(t)\right\|_{H}^{2}$ to both sides of (4.1), choose $\sigma$ small enough, and apply the generalized Gronwall lemma. Taking the infimum over all decompositions of $F_{\varepsilon}$ we have in view of.(3.21) and (2.31)

$$
\begin{aligned}
& \varepsilon^{2}\left\|\partial_{\ell}^{2} w_{\varepsilon}(t)\right\|_{H}^{2}+\varepsilon \iint_{Q_{!}}\left|\partial_{t}^{2} w_{\varepsilon}\right|^{2}+\varepsilon\left\|\partial_{t} w_{\varepsilon}(t)\right\|_{V}^{2} \\
& \leq c\left\|\chi_{\varepsilon}\right\|_{L^{\prime}(0, T ; W)}^{2} \\
& \quad+c\left(\varepsilon^{2}\left\|g_{\epsilon}(0)\right\|_{H}^{2}+\varepsilon\left\|\vartheta_{0, \varepsilon}+\ell \chi_{0, \varepsilon}\right\|_{V}^{2}+\varepsilon\left\|F_{\varepsilon}\right\|_{H^{\prime}(0, T ; H)+W^{2,1}\left(0, T ; V^{\prime}\right)}^{2}+1\right)
\end{aligned}
$$

$\leq c$. 
Let us introduce the notations

$$
\begin{aligned}
\hat{\chi}_{\varepsilon} & :=\chi_{\varepsilon}-\chi \\
\hat{\vartheta}_{\varepsilon} & :=\vartheta_{\varepsilon}-\vartheta \\
\hat{\eta}_{\varepsilon} & :=\left(\vartheta_{\varepsilon}+\ell \chi_{\varepsilon}\right)-(\vartheta+\ell \chi)=\hat{\vartheta}_{\varepsilon}+\ell \hat{\chi}_{\varepsilon} \\
u_{\varepsilon} & :=1 * \vartheta_{\varepsilon} \\
u & :=1 * \vartheta \\
\hat{u}_{\varepsilon} & :=u_{\varepsilon}-u \\
\hat{\xi}_{\varepsilon} & :=\xi_{\varepsilon}-\xi \\
\mu_{0, \varepsilon} & :=\frac{1}{|\Omega|} \int_{\Omega}\left(\chi_{0, \varepsilon}-\chi_{0}\right) .
\end{aligned}
$$

Note that the system described by the approximating problem is conserved, i.e. $\int_{\Omega} \chi_{\varepsilon}(t)$ $=\int_{\Omega} \chi_{0, \varepsilon}$ for all $t \in[0, T]$. We can pass to the limit here and get $\int_{\Omega} \chi(t)=\int_{\Omega} \chi_{0}$ for all $t \in[0, T]$. Owing to these two relations we have

$$
\mu_{0, \varepsilon}=\frac{1}{|\Omega|} \int_{\Omega}\left(\chi_{\varepsilon}-\chi\right)(t) \quad(t \in[0, T]) .
$$

In view of (2.17) and the definition of $u_{\varepsilon}$ we can rewrite (3.6) as

$$
\varepsilon \partial_{t}^{2} w_{\varepsilon}+\partial_{t} u_{\varepsilon}+k_{0} A u_{\varepsilon}=F_{\varepsilon}-\ell \chi_{\varepsilon}-A\left(\pi_{\varepsilon} * u_{\varepsilon}\right) .
$$

Integrating $(2.19)_{1}$ with respect to time we get

$$
\partial_{\imath} u+k_{0} A u=F-\ell \chi
$$

where $F$ is given by

$$
F=1 * g+\vartheta_{0}+\ell \chi_{0} \in H^{1}\left(0, T ; V^{\prime}\right)
$$

In order to estimate the term $\hat{u}_{\varepsilon}$, we take the difference between (4.4) and (4.j), i.e.

$$
\varepsilon \partial_{t}^{2} w_{\varepsilon}+\partial_{t} \hat{u}_{\varepsilon}+k_{0} A \hat{u}_{\varepsilon}=\left(F_{\varepsilon}-F\right)-\ell \hat{\chi}_{\varepsilon}-A\left(\pi_{\varepsilon} * u_{\varepsilon}\right)
$$

We test this equation by $\partial_{t} \hat{u}_{\varepsilon}$. As before, this formal procedure could be made rigorous using [6: Appendix]. Thus, after integration over $(0, t)$ we get

$$
\iint_{Q_{z}}\left|\partial_{t} \hat{u}_{\varepsilon}\right|+\frac{k_{0}}{2}\left\|\nabla \hat{u}_{\varepsilon}(t)\right\|_{H}^{2}=\sum_{i=1}^{4} \mathcal{I}_{i}(t)
$$

where

$$
\begin{aligned}
& \mathcal{I}_{1}(t)=\int_{0}^{t}\left\langle\left(F_{\varepsilon}-F\right)(s), \partial_{t} \hat{u}_{\epsilon}(s)\right\rangle d s \\
& \mathcal{I}_{2}(t)=-\varepsilon \iiint_{Q_{t}} \partial_{t}^{2} w_{\epsilon} \partial_{t} \hat{u}_{\varepsilon} \\
& \mathcal{I}_{3}(t)=-\ell \iint_{Q_{t}} \hat{\chi}_{\varepsilon} \partial_{t} \hat{u}_{\varepsilon} \\
& \mathcal{I}_{4}(t)=-\int_{0}^{t}\left\langle A\left(\pi_{e} * u_{\varepsilon}\right)(s), \partial_{t} \hat{u}_{\epsilon}(s)\right\rangle d s .
\end{aligned}
$$


Then we split

$$
F_{\varepsilon}-F=\Phi_{\varepsilon, 1}+\Phi_{\varepsilon, 2} \quad \text { with }\left\{\begin{array}{l}
\Phi_{\varepsilon, 1} \in L^{2}(0, T ; H) \\
\Phi_{\varepsilon, 2} \in H^{1}\left(0, T ; V^{\prime}\right)
\end{array}\right.
$$

and estimate $\mathcal{I}_{1}(t)$ after integration by parts as

$$
\begin{aligned}
\mathcal{I}_{1}(t)= & \iint_{Q_{t}} \Phi_{\varepsilon, 1} \partial_{t} \hat{u}_{\varepsilon}+\left\langle\Phi_{\varepsilon, 2}(t), \hat{u}_{\varepsilon}(t)\right\rangle-\int_{0}^{t}\left\langle\partial_{\ell} \Phi_{\varepsilon, 2}(s), \hat{u}_{\varepsilon}(s)\right\rangle d s \\
\leq & \sigma \iint_{Q_{t}}\left|\partial_{t} \hat{u}_{\varepsilon}\right|^{2}+c_{\sigma}\left\|\Phi_{\varepsilon, 1}\right\|_{L^{2}(0, T ; H)}^{2}+\sigma\left\|\hat{u}_{\varepsilon}(t)\right\|_{V}^{2} \\
& +c_{\sigma}\left\|\Phi_{\varepsilon, 2}\right\|_{H^{\prime}\left(0, T ; V^{\prime}\right)}^{2}+\frac{1}{2}\left\|\Phi_{\varepsilon, 2}\right\|_{H^{\prime}\left(0, T ; V^{\prime}\right)}^{2}+\frac{1}{2} \int_{0}^{t}\left\|\hat{u}_{\varepsilon}(s)\right\|_{V}^{2} d s .
\end{aligned}
$$

As far as $\mathcal{I}_{2}(t)$ and $\mathcal{I}_{3}(t)$ are concerned, we have obviously

$$
\begin{aligned}
& \mathcal{I}_{2}(t) \leq \sigma \iint_{Q_{t}}\left|\partial_{t} \hat{u}_{\varepsilon}\right|^{2}+c_{\sigma} \varepsilon^{2} \iint_{Q_{t}}\left|\partial_{t}^{2} w_{\varepsilon}\right|^{2} \\
& \mathcal{I}_{3}(t) \leq \sigma \iint_{Q_{t}}\left|\partial_{t} \hat{u}_{\varepsilon}\right|^{2}+c_{\sigma}\left\|\hat{\chi}_{\varepsilon}\right\|_{L^{2}(0, t ; H)}^{2} .
\end{aligned}
$$

To deal with $\mathcal{I}_{4}(t)$, we integrate by parts and use (2.1), (2.33), (2.35) and (3.10) to obtain

$$
\begin{aligned}
\mathcal{I}_{4}(t)= & -\left\langle A\left(\pi_{\varepsilon} * u_{\varepsilon}\right)(t), \hat{u}_{\varepsilon}(t)\right\rangle+\int_{0}^{t}\left\langle\partial_{t} A\left(\pi_{\varepsilon} * u_{\varepsilon}\right)(s), \hat{u}_{\varepsilon}(s)\right\rangle d s \\
= & -\int_{\Omega} \nabla\left(\dot{\pi}_{\varepsilon} * u_{\varepsilon}\right)(t) \cdot \nabla \hat{u}_{\varepsilon}(t)+\iint_{Q_{t}} \nabla\left(\pi_{\varepsilon}(0) u_{\varepsilon}+\pi_{\varepsilon}^{\prime} * u_{\varepsilon}\right) \cdot \nabla \hat{u}_{\varepsilon} \\
\leq & \sigma\left\|\nabla \hat{u}_{\varepsilon}(t)\right\|_{H}^{2}+c_{\sigma}\left\|\pi_{\varepsilon}\right\|_{L^{2}(0, T)}^{2} \iint_{Q_{t}}\left|\nabla u_{\varepsilon}\right|^{2} \\
& +\iint_{Q_{t}}\left|\nabla \hat{u}_{\varepsilon}\right|^{2}+c\left(\left|\pi_{\varepsilon}(0)\right|^{2}+\left\|\pi_{\varepsilon}^{\prime}\right\|_{L^{1}(0, T)}^{2}\right) \iint_{Q_{t}}\left|\nabla u_{\varepsilon}\right|^{2} \\
\leq & \sigma\left\|\nabla \hat{u}_{\varepsilon}(t)\right\|_{H}^{2}+c_{\sigma}\left\|\pi_{\varepsilon}\right\|_{W^{1,1}(0, T)}^{2} \iint_{Q_{t}}\left|\nabla u_{\varepsilon}\right|^{2}+\iint_{Q_{t}}\left|\nabla \hat{u}_{\varepsilon}\right|^{2} \\
\leq & \sigma\left\|\nabla \hat{u}_{\varepsilon}(t)\right\|_{H}^{2}+c_{\sigma}\left\|\pi_{\varepsilon}\right\|_{W^{1,1}(0, T)}^{2}+\iint_{Q_{t}}\left|\nabla \hat{u}_{\varepsilon}\right|^{2} .
\end{aligned}
$$

Adding $\frac{k_{0}}{2}\left\|\hat{u}_{\epsilon}(t)\right\|_{H}^{2}$ to both sides of (4.6) and observing that

$$
\frac{k_{0}}{2}\left\|\hat{u}_{\varepsilon}(t)\right\|_{H}^{2} \leq c \int_{0}^{t}\left\|\hat{u}_{\varepsilon}(s)\right\|_{H}\left\|\partial_{t} \hat{u}_{\varepsilon}(s)\right\|_{H} d s \leq \sigma \iint_{Q_{t}}\left|\partial_{t} \hat{u}_{\varepsilon}\right|^{2}+c_{\sigma} \iint_{Q_{t}}\left|\hat{u}_{\varepsilon}\right|^{2}
$$


we obtain, after choosing $\sigma$ small enough,

$$
\begin{aligned}
& \iint_{Q_{t}}\left|\partial_{t} \hat{u}_{\varepsilon}\right|^{2}+\left\|\hat{u}_{\varepsilon}(t)\right\|_{V}^{2} \\
& \leq c\left\|\Phi_{\varepsilon, 1}\right\|_{L^{2}(0, T ; H)}^{2}+c\left\|\Phi_{\varepsilon, 2}\right\|_{H^{1}\left(0, T ; V^{\prime}\right)}^{2} \\
& \quad+c \varepsilon^{2} \iint_{Q_{t}}\left|\partial_{\ell}^{2} w_{\varepsilon}\right|^{2}+c\left\|\hat{\chi}_{\epsilon}\right\|_{L^{2}(0, t ; H)}^{2}+c\left\|\pi_{\varepsilon}\right\|_{W^{1,1}(0, T)}^{2} \\
& \quad+c \int_{0}^{t}\left\|\hat{u}_{\varepsilon}(s)\right\|_{V}^{2} d s+c \int_{0}^{t}\left\|\hat{u}_{\epsilon}(s)\right\|_{V}^{2} d s
\end{aligned}
$$

Now we can apply the Gronwall lemma and, taking the infimum over all decompositions $F_{\varepsilon}-F=\Phi_{\varepsilon, 1}+\Phi_{\varepsilon, 2}$ we get

$$
\begin{aligned}
\iint_{Q_{t}}\left|\partial_{t} \hat{u}_{\epsilon}\right|^{2}+\left\|\hat{u}_{\varepsilon}(t)\right\|_{V}^{2} \leq & c\left(\varepsilon^{2}\left\|\partial_{\imath}^{2} w_{\varepsilon}\right\|_{L^{2}(0, T ; H)}^{2}\right. \\
& +\left\|F_{\varepsilon}-F\right\|_{L^{2}(0, T ; H)+H^{1}\left(0, T ; V^{\prime}\right)}^{2} \\
& \left.+\left\|\hat{\chi}_{\varepsilon}\right\|_{L^{2}(0, t ; H)}^{2}+\left\|\pi_{\varepsilon}\right\|_{W^{1,1}(0, T)}^{2}\right)
\end{aligned}
$$

Taking, the difference between $(2.16)_{2}$ and $(2.19)_{2}$ we get $\partial_{i} \hat{\chi}_{\varepsilon}+A \hat{\xi}_{\varepsilon}=0$. Owing to (4.3), we have $\hat{\chi}_{\varepsilon}(t)-\mu_{0, \varepsilon} \in \mathcal{V}$. Hence $\mathcal{N}\left(\hat{\chi}_{\varepsilon}(t)-\mu_{0, \varepsilon}\right)$ is an admissible test function for the previous equation and, in view of $(2.2)$, we get

$$
\frac{1}{2} \frac{d}{d t} \int_{\Omega}\left|\nabla \mathcal{N}\left(\hat{\chi}_{\varepsilon}(t)-\mu_{0, \varepsilon}\right)\right|^{2}+\left\langle\hat{\chi}_{\varepsilon}(t)-\mu_{0, \varepsilon}, \hat{\xi}_{\varepsilon}(t)\right\rangle=0 .
$$

Thanks to $(2.18)_{3}$ and $(2.21)_{3}$, the second term is given by

$$
\begin{aligned}
\left\langle\hat{\chi}_{\varepsilon}(t)\right. & \left.-\mu_{0, \varepsilon}, \hat{\xi}_{\varepsilon}(t)\right\rangle \\
= & \left\langle\hat{\chi}_{\varepsilon}(t)-\mu_{0, \varepsilon},\left(A \hat{\chi}_{\varepsilon}+\chi_{\varepsilon}^{3}-\chi^{3}+\left(\ell^{2}-1\right) \hat{\chi}_{\varepsilon}-\ell \hat{\eta}_{\varepsilon}\right)(t)\right\rangle \\
= & \int_{\Omega}\left|\nabla\left(\hat{\chi}_{\varepsilon}(t)-\mu_{0, \varepsilon}\right)\right|^{2}+\int_{\Omega}\left(\left(\chi_{\varepsilon}^{3}-\chi^{3}\right) \hat{\chi}_{\varepsilon}\right)(t)-\mu_{0, \varepsilon} \int_{\Omega}\left(\chi_{\varepsilon}^{3}-\chi^{3}\right)(t) \\
& +\left(\ell^{2}-1\right) \int_{\Omega}\left(\hat{\chi}_{\varepsilon}(t)-\mu_{0, \varepsilon}\right) \hat{\chi}_{\varepsilon}(t)-\ell\left\langle\hat{\eta}_{\varepsilon}(t), \hat{\chi}_{\varepsilon}(t)-\mu_{0, \varepsilon}\right\rangle .
\end{aligned}
$$

After noting that

$$
\int_{\Omega}\left(\hat{\chi}_{\varepsilon}(t)-\mu_{0, \varepsilon}\right) \hat{\chi}_{\varepsilon}(t)=\int_{\Omega}\left(\hat{\chi}_{\varepsilon}(t)-\mu_{0, \epsilon}\right)^{2}
$$

we can deduce

$$
\begin{aligned}
& \frac{1}{2} \frac{d}{d t} \int_{\Omega}\left|\nabla \mathcal{N}\left(\hat{\chi}_{e}(t)-\mu_{0, \varepsilon}\right)\right|^{2}+\int_{\Omega}\left|\nabla\left(\bar{\chi}_{e}(t)-\mu_{0, e}\right)\right|^{2} \\
& \quad+\int_{\Omega}\left(\left(\chi_{\varepsilon}^{3}-\chi^{3}\right) \hat{\chi}_{e}\right)(t)+\ell^{2}\left\|\hat{\chi}_{e}(t)-\mu_{0, \varepsilon}\right\|_{H}^{2} \\
& =\mu_{0, \varepsilon} \int_{\Omega}\left(\chi_{\varepsilon}^{3}-\chi^{3}\right)(t)+\left\langle\hat{\chi}_{\varepsilon}(t)-\mu_{0, \varepsilon}+\ell \hat{\eta}_{\varepsilon}(t), \hat{\chi}_{\varepsilon}(t)-\mu_{0, \epsilon}\right\rangle .
\end{aligned}
$$


Estimating the last term by

$$
c_{\sigma}\left\|\left(\hat{\chi}_{\varepsilon}-\mu_{0, \varepsilon}+\ell \hat{\eta}_{\varepsilon}\right)(t)\right\|_{V^{\prime}}^{2}+\sigma\left\|\hat{\chi}_{\varepsilon}(t)-\mu_{0, \varepsilon}\right\|_{V}^{2}
$$

thanks to (2.35), recalling (2.3), choosing $\sigma=\frac{1}{2} \min \left\{1, \ell^{2}\right\}$ and integrating over $(0, t)$, it is easy to obtain

$$
\begin{aligned}
\frac{1}{2} \| \hat{\chi}_{\epsilon}(t) & -\mu_{0, \varepsilon}\left\|_{V^{\prime}}^{2}+\frac{1}{2} \min \left\{1, \ell^{2}\right\} \int_{0}^{l}\right\| \hat{\chi}_{\varepsilon}(s)-\mu_{0, \varepsilon} \|_{V^{2}}^{2} d s \\
\leq & \frac{1}{2}\left\|\chi_{0, \varepsilon}-\chi_{0}-\mu_{0, \varepsilon}\right\|_{V^{\prime}}^{2}+\mu_{0, \varepsilon} \iint_{Q_{\varepsilon}}\left(\chi_{\varepsilon}^{3}-\chi^{3}\right) \\
& +c \int_{0}^{\ell}\left(\left\|\left(\hat{\chi}_{\varepsilon}-\mu_{0, \varepsilon}\right)(s)\right\|_{V^{\prime}}^{2}+\left\|\hat{\eta}_{\varepsilon}(s)\right\|_{V^{\prime}}^{2}\right) d s
\end{aligned}
$$

where the monotonicity of the function $x \mapsto x^{3}$ has been used.

Note that $\hat{\eta}_{\varepsilon}=\partial_{t} \hat{u}_{\varepsilon}+\ell \hat{\chi}_{\varepsilon}$, whence

$$
\begin{aligned}
\left\|\hat{\eta}_{\varepsilon}(s)\right\|_{V^{\prime}}^{2} & \leq c\left(\left\|\partial_{t} \hat{u}_{\varepsilon}(s)\right\|_{V^{\prime}}^{2}+\left\|\hat{\chi}_{\varepsilon}(s)\right\|_{V^{\prime}}^{2}\right) \\
& \leq c\left(\left\|\partial_{t} \hat{u}_{\varepsilon}(s)\right\|_{V^{\prime}}^{2}+\left\|\hat{\chi}_{\varepsilon}(s)-\mu_{0, \varepsilon}\right\|_{V^{\prime}}^{2}+\mu_{0, \varepsilon}^{2}\right) .
\end{aligned}
$$

Then we have

$$
\begin{aligned}
& \left\|\hat{\chi}_{\varepsilon}(t)-\mu_{0, \varepsilon}\right\|_{V^{\prime}}^{2}+\left\|\hat{\chi}_{\varepsilon}-\mu_{0, \varepsilon}\right\|_{L^{2}(0, t ; V)}^{2} \\
& \leq c\left\|\chi_{0, \varepsilon}-\chi_{0}-\mu_{0, \varepsilon}\right\|_{V^{\prime}}^{2}+c \mu_{0, \varepsilon} \iint_{Q_{t}}\left(\chi_{\varepsilon}^{3}-\chi^{3}\right) \\
& \quad+c \int_{0}^{t}\left\|\hat{\chi}_{\varepsilon}(s)-\mu_{0, \varepsilon}\right\|_{V^{\prime}}^{2} d s+c \mu_{0, \varepsilon}^{2}+c \int_{0}^{t}\left\|\partial_{t} \hat{u}_{\varepsilon}(s)\right\|_{V^{\prime}}^{2} d s .
\end{aligned}
$$

Note that

$$
\begin{aligned}
& c \mu_{0, \varepsilon} \iint_{Q_{t}}\left(\chi_{\varepsilon}^{3}-\chi^{3}\right) \\
& \quad=c \mu_{0, \varepsilon} \iint_{Q_{t}}\left(\hat{\chi}_{\varepsilon}-\mu_{0, \varepsilon}\right)\left(\chi_{\varepsilon}^{2}+\chi^{2}+\chi \chi_{\varepsilon}\right)+c \mu_{0, \varepsilon}^{2} \iint_{Q_{t}}\left(\chi_{\varepsilon}^{2}+\chi^{2}+\chi \chi_{\varepsilon}\right) \\
& \quad \leq 2 c\left|\mu_{0, \varepsilon}\right| \iint_{Q_{t}}\left|\hat{\chi}_{\varepsilon}-\mu_{0, \varepsilon}\right|\left(\chi_{\varepsilon}^{2}+\chi^{2}\right)+2 c \mu_{0, \varepsilon}^{2} \iint_{Q_{t}}\left(\chi_{\varepsilon}^{2}+\chi^{2}\right) . \\
& \quad \leq \frac{1}{2}\left\|\hat{\chi}_{\varepsilon}-\mu_{0, \varepsilon}\right\|_{L^{2}\left(0, t_{;} H\right)}^{2}+c \mu_{0, \varepsilon}^{2}\left(\left\|\chi_{\varepsilon}\right\|_{L^{4}(\Omega \times(0, T))}^{4}+\|\chi\|_{L^{4}(\Omega \times(0, T))}^{4}\right) .
\end{aligned}
$$

Thanks to (4.9) and (4.7), (4.8) yields

$$
\begin{aligned}
& \left\|\hat{\chi}_{\varepsilon}(t)-\mu_{0, \varepsilon}\right\|_{V^{\prime}}^{2}+\left\|\hat{\chi}_{\varepsilon}-\mu_{0, \varepsilon}\right\|_{L^{2}(0, t ; V)}^{2} \\
& \leq c\left\|\chi_{0, \varepsilon}-\chi_{0}-\mu_{0, \varepsilon}\right\|_{V^{\prime}}^{2}+\frac{1}{2}\left\|\hat{\chi}_{\varepsilon}-\mu_{0, \varepsilon}\right\|_{L^{2}(0, t ; H)}^{2} \\
& \quad+c \mu_{0, \varepsilon}^{2}\left(\left\|\chi_{\varepsilon}\right\|_{L^{4}\left(\Omega \times\left(0, T^{\prime}\right)\right)}^{4}+\|\chi\|_{L^{4}(\Omega \times(0, T))}^{4}\right) \\
& \quad+c \int_{0}^{\ell}\left\|\hat{\chi}_{\varepsilon}(s)-\mu_{0, \varepsilon}\right\|_{V^{\prime}}^{2} d s \\
& \quad+c \mu_{0, \varepsilon}^{2}+c\left(\left\|F_{\varepsilon}-F\right\|_{L^{2}(0, T ; H)+H^{1}\left(0, T ; V^{\prime}\right)}^{2}+\varepsilon^{2}\left\|\partial_{t}^{2} w_{\varepsilon}\right\|_{L^{2}\left(0, T_{;}, H\right)}^{2}+\left\|\pi_{\varepsilon}\right\|_{W^{1,1}(0, T)}^{2}\right) \\
& \quad+c\left\|\hat{\chi}_{\varepsilon}-\mu_{0, \varepsilon}\right\|_{L^{2}(0, t ; H)}^{2} \cdot
\end{aligned}
$$


Note that, since $\left(\hat{\chi}_{\varepsilon}-\mu_{0, \varepsilon}\right)(t) \in V$;

$$
\begin{aligned}
\left\|\hat{\chi}_{\varepsilon}-\mu_{0, \varepsilon}\right\|_{L^{2}(0, t ; H)}^{2} & =\int_{0}^{t}\left\langle\left(\hat{\chi}_{\varepsilon}-\mu_{0, \varepsilon}\right)(s),\left(\hat{\chi}_{\varepsilon}-\mu_{0, \epsilon}\right)(s)\right\rangle d s \\
& \leq \frac{1}{4 \sigma} \int_{0}^{\ell}\left\|\hat{\chi}_{\varepsilon}(s)-\mu_{0, \varepsilon}\right\|_{V^{\prime}}^{2} d s+\sigma \int_{0}^{\ell}\left\|\hat{\chi}_{\varepsilon}(s)-\mu_{0, \varepsilon}\right\|_{V}^{2} d s .
\end{aligned}
$$

Hence, choosing $\sigma$ small enough and recalling $(3.14)_{3}$, we have

$$
\begin{aligned}
& \left\|\hat{\chi}_{\varepsilon}(t)-\mu_{0, \varepsilon}\right\|_{V^{\prime}}^{2}+\left\|\hat{\chi}_{\varepsilon}-\mu_{0, \varepsilon}\right\|_{L^{2}(0, t ; V)}^{2} \\
& \quad \leq c\left\|\chi_{0, \varepsilon}-\chi_{0}-\mu_{0, \varepsilon}\right\|_{V^{\prime}}^{2}+c \int_{0}^{t}\left\|\hat{\chi}_{\varepsilon}(s)-\mu_{0, \varepsilon}\right\|_{V^{\prime}}^{2} d s+c \mu_{0, \epsilon}^{2} . \\
& \quad+c\left(\left\|F_{\varepsilon}-F\right\|_{L^{2}(0, T ; H)+H^{1}\left(0, T ; V^{\prime}\right)}^{2}+\varepsilon^{2}\left\|\partial_{t}^{2} w_{\varepsilon}\right\|_{L^{2}(0, T ; H)}^{2}+\left\|\pi_{\varepsilon}\right\|_{W^{1,1}(0, T)}^{2}\right) .
\end{aligned}
$$

Applying the Gronwall lemma and recalling (4.2) we have

$$
\begin{aligned}
& \left\|\hat{\chi}_{\epsilon}(t)-\mu_{0, \varepsilon}\right\|_{V^{\prime}}^{2}+\left\|\hat{\chi}_{\varepsilon}-\mu_{0, \varepsilon}\right\|_{L^{2}\left(0, t^{\prime} V\right)}^{2} \\
& \quad \leq c\left(\mu_{0, \varepsilon}^{2}+\left\|\chi_{0, \varepsilon}-\chi_{0}\right\|_{V^{\prime}}^{2}+\left\|F_{\varepsilon}-F\right\|_{L^{2}(0, T ; H)+H^{1}\left(0, T ; V^{\prime}\right)}^{2}+\left\|\pi_{\varepsilon}\right\|_{W^{1,1}(0, T)}^{2}\right)+c \varepsilon
\end{aligned}
$$

which implies

$$
\begin{aligned}
& \left\|\hat{\chi}_{\varepsilon}\right\|_{L^{\infty}\left(0, T ; V^{\prime}\right) \cap L^{2}(0, T ; V)} \\
& \leq c\left(\left|\mu_{0, \varepsilon}\right|+\left\|\chi_{0, \varepsilon}-\chi_{0}\right\| V^{\prime}+\left\|F_{\varepsilon}-F\right\|_{L^{2}(0, T ; H)+H^{1}\left(0, T ; V^{\prime}\right)}+\left\|\pi_{\varepsilon}\right\|_{W^{1,1}(0, T)}\right) \\
& \quad+c \varepsilon^{\frac{1}{2}}
\end{aligned}
$$

Then (4.7) and (4.10) yield (2.32), thanks to (4.2) and (2.31)

Remark. The techniques used in the previous error estimate are similar to those cmployed in the proof of the continuous dependence of [9: Lemma 3.1].

\section{References}

[1] Baiocchi, C.: Soluzioni ordinarie e generalizzate del problema di Cauchy per equazioni differenziali astratte lineari del secondo ordine in spazi di Hilbert. Ricerche Mat. 16 (1967), $27-95$.

[2] Brezis, H.: Opérateurs maximaux monotones et semi-groupes de contractions dans les espaces de Hilbert (North-Holland Math. Stud.: Vol. 5). Amsterdam: North-Holland 1973.

[3] Brochet, D., Hilhorst, D. and A. Novick-Cohen: Maximal attractor and inertial sets for a conserved phase field model. Adv. Diff. Equ. 1 (1996), $547-578$.

[4] Cattaneo, C.: Sulla conduzione del calore. Atti Sem. Mat. Fis. Univ. Modena 3 (1948), $83-101$. 
[5] Colli, P., Gilardi, G. and M. Grasselli: Global smooth solution to the standard phase-field model with memory. $\Lambda \mathrm{dv}$. Diff. Equ. 2 (1997), $453-486$.

[6] Colli, P., Gilardi, G. and M. Grasselli: Well-posedness of the weak formulation for the phase-field model with memory. Adv. Diff. Equ. 2 (1997), 487 - 508.

[7] Colli, P., Gilardi, G. and M. Grasselli: Asymptotic justification of the phase-field model with memory. Comm. Appl. Nonlin. Anal. (to appear).

[8] Colli, P., Gilardi, G., Grasselli, M. and G. Schimperna: The conserved phase-field system with memory. Preprint. Politecnico Milano: Preprint 376/P (1999).

[9] Colli, P., Gilardi, G., Laurençot, Ph. and A. Novick-Cohen: Uniqueness and long-time behavior for the conserved phase-field system with memory. Discrete Contin. Dynam. Systems 5 (1999); 375 - 390.

[10] Felli, V.: Convergenza al modello classico di un problema di campo di fase conservativo con memoria. Graduation thesis. Pavia 1999.

[11] Gurtin, M. E. and A. C. Pipkin: A general theory of heat conduction with finite wave speeds. Arch. Rat. Mech. Anal. 31 (1968), $113-126$.

[12] Novick-Cohen, A.: Conserved phase-field equations with memory. In: Curvature flows and related topics (eds.: A. Damlamian et al.; GAKUTO Int. Ser. Math. Sci. Appl.: Vol. 5). Tokyo: Gakkōtosho 1995, pp. $179-197$.

[13] Schwartz, L.: Théorie des distributions. Paris: Hermann 1966.

[14] Simon, J.: Compact sets in the space $L^{P}(0, T ; B)$. Ann. Mat. Pura Appl. (4) 146 (1987), $65-96$.

Received 04.01.2000, in revised form 16.05.2000 